Research Article

\title{
Analysis of the Mechanical Behaviour of Asphalt Concretes Using Artificial Neural Networks
}

\author{
Nicola Baldo iD, $^{1}$ Evangelos Manthos, ${ }^{2}$ and Marco Pasetto (iD $^{3}$ \\ ${ }^{1}$ Polytechnic Department of Engineering and Architecture, University of Udine, Via del Cotonificio 114, 33100 Udine, Italy \\ ${ }^{2}$ Department of Civil Engineering, Aristotle University of Thessaloniki, University Campus, 54124 Thessaloniki, Greece \\ ${ }^{3}$ Department of Civil, Environmental and Architectural Engineering, University of Padua, Via Marzolo 9, 35131 Padua, Italy
}

Correspondence should be addressed to Nicola Baldo; nicola.baldo@uniud.it

Received 27 February 2018; Revised 12 June 2018; Accepted 19 June 2018; Published 12 July 2018

Academic Editor: Victor Yepes

Copyright ( $\odot 2018$ Nicola Baldo et al. This is an open access article distributed under the Creative Commons Attribution License, which permits unrestricted use, distribution, and reproduction in any medium, provided the original work is properly cited.

\begin{abstract}
The current paper deals with the numerical prediction of the mechanical response of asphalt concretes for road pavements, using artificial neural networks (ANNs). The asphalt concrete mixes considered in this study have been prepared with a diabase aggregate skeleton and two different types of bitumen, namely, a conventional bituminous binder and a polymer-modified one. The asphalt concretes were produced both in a road materials laboratory and in an asphalt concrete production plant. The mechanical behaviour of the mixes was investigated in terms of Marshall stability, flow, quotient, and moreover by the stiffness modulus. The artificial neural networks used for the numerical analysis of the experimental data, of the feedforward type, were characterized by one hidden layer and 10 artificial neurons. The results have been extremely satisfactory, with coefficients of correlation in the testing phase within the range $0.98798-0.91024$, depending on the considered model, thus demonstrating the feasibility to apply ANN modelization to predict the mechanical and performance response of the asphalt concretes investigated. Furthermore, a closed-form equation has been provided for each of the four ANN models developed, assuming as input parameters the production process, the bitumen type and content, the filler/bitumen ratio, and the volumetric properties of the mixes. Such equations allow any other researcher to predict the mechanical parameter of interest, within the framework of the present study.
\end{abstract}

\section{Introduction}

In order to design a road superstructure, the so-called pavement, two main tasks have to be accomplished, namely, the mix design of the asphalt concrete to be used for each of the layers of the pavement and the thickness design of the pavement itself. Focusing the attention on the mix design task, currently, all over the world, experimental procedures carried out in a road laboratory are adopted [1-6]. Actually, a preliminary material characterization has to be performed for both the asphalt concrete components, namely, aggregates and bitumen, as well as a proper experimental mix design procedure is required in order to identify the optimum bitumen content. The laboratory tests used to evaluate the physical properties and the mechanical resistance of components and mixtures are quite time consuming; moreover, skilled laboratory technicians have to be involved. At the end of the experimental mix design procedure, the best suited formulation of the asphalt mix is identified, in order to meet the pavement service requirements. However, on the basis of a pure experimental approach, if a component type or its amount has to be modified, for instance, for comparison purposes between different materials, to identify the best technological solution for the pavement construction, further laboratory tests cannot be avoided in order to evaluate the different mechanical response of the asphalt mix. The possibility to estimate the mechanical behaviour of the mix, on the basis of a mathematical model of the material's response, would allow us to save time and cost of further experiments.

A material's response model can be elaborated by means of constitutive equations [7-9] implemented on a computational platform with the finite element method [10-12], rather than with the discrete element method [13-15]. Such approaches are elaborated on a physical basis because they try to 
achieve a rational interpretation of the mechanical response of the asphalt concrete under different loading conditions. However, the complexity of such methods is quite high, being related to the proper formulation of the constitutive equations as well as to extensive laboratory trials (which often involve particular test protocols), aimed at properly calibrating and validating such complex mathematical models. A different approach is based on the statistical regression of large experimental data sets, to obtain prediction equations of the material properties considered [16-19].

More recently, the so-called learning machines, for instance, the artificial neural networks (ANNs), have been used for the modelization of some significant mechanical parameters of road materials [20-23]. The key point of such approaches is given by the possibility to obtain reliable analytical equations for the quantitative estimation of materials properties, in a relatively automatized and easy way, because the complex physical significance of the material's response is not considered; such an advantage, the so-called "black box esffect," represents on the contrary their main issue. The abovementioned black box effect could somehow be associated also with the relatively low attention paid in the civil engineering literature to the mathematical equations behind the artificial neural networks. Actually, several literature papers are simply devoted to the use of such computational tools in a broad variety of engineering applications, but without a proper discussion of the mathematical framework [24-29]. Moreover, in such papers, the discussion is often limited to the evaluation of the quality of the training and testing phases of the ANN; just few researchers $[20,30,31]$ have at least presented the predictive analytical equations elaborated by means of the ANN.

The main goal of this paper is to provide the analytical expressions for the prediction of the mechanical parameters involved in the mix design of asphalt concretes, on the basis of the ANN modelization of experimental data related to volumetric and composition parameters of the mixes. In order to allow a full understanding of the ANN modelization, a theoretical discussion of the mathematical equations, which actually constitute the backbone of the ANN, is also provided.

\section{Theory and Calculation}

2.1. Modelling with Artificial Neural Networks. Artificial neural networks (ANNs), also known as learning machines, represent a computational approach to develop predictive models for the desired parameter, whatever the complexity of the system under investigation, given a robust experimental data set for the training of the ANN [32]. They try to simulate the functioning of biological ones, in particular those of the brain, processing the input data through "neurons" [33-35].

There are different types of ANN; in this study, the feedforward networks have been considered. For such ANN type, the learning of the network is supervised; therefore, for each input vector provided, the corresponding output vector (target) is known. The learning phase consists in optimizing the connections of the ANN so that for each input considered, the network returns a calculated value as close as possible to the target one [35].
An ANN of this type is structured with different neurons, divided into layers; these neurons are connected so that those of the same layer are not linked to each other and that none of the possible paths could touch twice the same neuron. Therefore, the feedforward ANN structure is given by the following:

(i) An initial layer with $p$ neurons (input layer), where $p$ is the number of input variables

(ii) A final layer of $c$ neurons (output layer), where $c$ is the number of output variables

(iii) At least one intermediate layer, or hidden layer, with $m$ number of neurons, independent of how many belong to input or output

The input layer stores the incoming signals that are introduced through a vector $\overline{\mathbf{x}}_{\mathbf{i}}$ for each data set:

$$
\overline{\mathbf{x}}_{\mathbf{i}}=\left(x_{1 i}, x_{2 i}, \ldots, x_{p i}\right), \quad i=1, \ldots, n .
$$

The output layer provides the calculated values through a vector $\overline{\mathbf{y}}_{\mathbf{i}}$ :

$$
\overline{\mathbf{y}}_{\mathbf{i}}=\left(y_{1 i}, y_{2 i}, \ldots, y_{c i}\right), \quad i=1, \ldots, n .
$$

The hidden layer is devoted to the calculations that formally connect the input $\overline{\mathbf{x}}_{\mathbf{i}}$ with the output $\overline{\mathbf{y}}_{\mathbf{i}}$.

Each neuron of the hidden layer works according to a simple mathematical model proposed by McCulloch and Pitts [36]. A weighted sum of the values of the input variables is computed through the weights that are associated with each connection:

$$
a=\sum_{i=1}^{p} w_{i} x_{i}+w_{0}
$$

The value $w_{0}$ is called bias and corresponds to the activation value of the neuron; assuming $x_{0}=1$, such an expression can be simplified as follows:

$$
a=\sum_{i=0}^{p} w_{i} x_{i}
$$

The output value from the neuron is calculated by applying an appropriate transfer function to such a value:

$$
z=f(a)=f\left(\sum_{i=0}^{p} w_{i} x_{i}\right) .
$$

The transfer function can be of different types depending on the desired model, for instance, linear, Heaviside step function, sigmoidal, or hyperbolic tangent. The last one has been used in the current study; it has the following expression:

$$
f(a)=\frac{e^{a}-e^{-a}}{e^{a}+e^{-a}}=\frac{2}{1+e^{-2 a}}-1 .
$$

The above steps describe the functioning of a single neuron, while the network, to determine the optimal values of the weights of each connection, follows an iterative procedure, the so-called training of the neural network [33-35]. Given a set of first attempt values of the weights, the ANN computes the activation values of the neurons and 
subsequently the final output; this phase is known as the forward pass. Then, the value of the calculated output is compared with the expected value (target) so that the ANN can proceed to adjust the weights through an optimization algorithm; this phase is called backward pass.

2.2. The Forward Pass. Considering an ANN with $p$ neurons in input, a single hidden layer with $m$ neurons, and an output layer with $c$ neurons, the network processes the information based on the procedure described in the following.

For each of the $i$ observations, or data sets $\left(\overline{\mathbf{x}}_{\mathbf{i}}\right)$, whose $p$ coordinates are introduced each one in a neuron of the input layer, the activation value of each neuron $j$ of the hidden layer is calculated:

$$
a_{j}^{(1)}=a_{j}^{(1)}\left(\overline{\mathbf{x}}_{\mathbf{i}}\right)=w_{o j}^{(1)}+\sum_{s=1}^{p} w_{s j}^{(1)} x_{i s},
$$

where the exponent (1) identifies the weights and the activation value of the first step, that is, between the input layer and the hidden one. Introducing again a fictitious value $x_{0 i}=1$, it follows that

$$
a_{j}^{(1)}=a_{j}^{(1)}\left(\overline{\mathbf{x}}_{\mathbf{i}}\right)=\sum_{s=0}^{p} w_{s j}^{(1)} x_{i s} .
$$

For each $\left(\overline{\mathbf{x}}_{\mathbf{i}}\right)$, the activation values $a_{j}^{(1)}$ are then transferred to the next level through a transfer function $f$ :

$$
z_{k}=f\left(a_{j}^{(1)}\right) \text {. }
$$

The procedure is repeated between the hidden layer and the output layer by calculating the activation value for each of the output $c$ neurons as follows:

$$
a_{k}^{(2)}=a_{k}^{(2)}\left(\overline{\mathbf{x}}_{\mathbf{i}}\right)=\sum_{j=0}^{m} w_{j k}^{(2)} z_{k}=\sum_{j=0}^{m} w_{j k}^{(2)} f\left(a_{j}^{(1)}\right),
$$

where the exponent (2) identifies the weights and the activation value of the second step, having made an assumption similar to the first step, that is, $f\left(a_{j}^{(1)}\right)=1$.

The output of the network introduces a further transfer through a function $g$, not necessarily of the same type of $f$; therefore, the calculated output value at the final $k$ th neuron is

$$
y_{i k}=g\left(a_{k}^{(2)}\right)=g\left[\sum_{j=0}^{m} w_{j k}^{(2)} f\left(\sum_{s=0}^{p} w_{s j}^{(1)} x_{i s}\right)\right] .
$$

2.3. The Backward Pass. The ANN proceeds updating the weights of the connections that are the only modifiable parameters, in fact the values of the components of each $\left(\overline{\mathbf{x}}_{\mathbf{i}}\right)$ are fixed; therefore, the interpolating function depends only on the weights of the individual connections. These can be considered as the parameters of an interpolating function for the approximation of the target values $t_{i k}$ with the computed values $y_{i k}$; the optimal value of these weights is then calculated minimizing the objective function:

$$
E=\frac{1}{2} \sum_{i=1}^{n} \sum_{k=1}^{c}\left(y_{i k}-t_{i k}\right)^{2} .
$$

Since the $t_{i k}$ values are given, this objective function depends only on the weights of the single connections. The weight vector $\mathbf{w}$ therefore, given the complexity of the function $E$, is calculated iteratively through an optimization algorithm [33-35]; the simplest one is the gradient descent algorithm. At each iteration, the weight vector is updated according to the following equation:

$$
\overline{\mathbf{w}}_{\tau}=\overline{\mathbf{w}}_{\boldsymbol{\tau}-1}+\overline{\Delta \mathbf{w}},
$$

where the subscript $\tau$ indicates the number of the iteration and the quantity $\overline{\Delta \mathbf{w}}$, which updates the weights, is a vector that moves along the descending path, characterized by a faster reduction of the function $E$, that is, its gradient in the vector space generated by the weights. Therefore, it can be written as follows:

$$
\overline{\Delta \mathbf{w}}=-\eta \nabla\left(E\left(\overline{\mathbf{w}}_{\tau}\right)\right) \Rightarrow \overline{\mathbf{w}}_{\boldsymbol{\tau}}=\overline{\mathbf{w}}_{\boldsymbol{\tau}-1}-\eta \nabla\left(E\left(\overline{\mathbf{w}}_{\tau}\right)\right) .
$$

To find this direction, it is necessary to compute the partial derivatives of the objective function and to define the value of $\eta$ which is a positive real number that should not be too small; otherwise, the calculation time becomes longer, or too big, to avoid the instability of the method.

Specifying the structure of the function $E$, it can be written as follows:

$$
E=\sum_{i=1}^{n} E_{i} \Rightarrow E_{i}=\frac{1}{2} \sum_{k=1}^{c}\left(y_{i k}-t_{i k}\right)^{2} .
$$

Thus, the partial derivatives can be calculated for each $E_{i}$ and subsequently added together; basically each $E_{i}$ can be considered as the component of a vector. The partial derivatives must be expressed with respect to the weights, and these are relative to both the hidden and output layers. Considering the partial derivatives of the output layer, if its transfer function is of the linear type, as it has been assumed in the present study, it follows that

$$
y_{i k}=g\left(a_{k}^{(2)}\right)=a_{k}^{(2)}=\sum_{j=0}^{m} w_{j k}^{(2)} f\left(a_{j}^{(1)}\right) .
$$

Therefore, the partial derivative of the generic term of $E_{i}$ with respect to the generic weight $w_{j k}^{(2)}$ results

$$
\frac{\partial E_{i}}{\partial w_{j k}^{(2)}}=\left(y_{i k}-t_{i k}\right) \frac{\partial y_{i k}}{\partial w_{j k}^{(2)}}=\left(y_{i k}-t_{i k}\right) f\left(a_{j}^{(1)}\right),
$$

where $a_{j}^{(1)}=\sum_{s=0}^{p} w_{s j}^{(1)} x_{i s}$ and $f$ is the transfer function of the hidden layer. In the present study, the hyperbolic tangent function has been assumed for the hidden layer:

$$
f\left(a_{j}^{(1)}\right)=\frac{2}{1+e^{-2 a_{j}^{(1)}}}-1=\frac{2}{1+e^{-2 \sum_{s=0}^{p} w_{s j}^{(1)} x_{i s}}}-1 .
$$

Instead, deriving $E_{i}$ with respect to a weight of the hidden layer and using the chain rule, it can be written as follows: 


$$
\frac{\partial E_{i}}{\partial w_{s j}^{(1)}}=\sum_{k=1}^{c}\left(y_{i k}-t_{i k}\right) \frac{\partial y_{i k}}{\partial w_{s j}^{(1)}}=\sum_{k=1}^{c}\left(y_{i k}-t_{i k}\right) \frac{\partial y_{i k}}{\partial a_{j}^{(1)}} \frac{\partial a_{j}^{(1)}}{\partial w_{s j}^{(1)}}
$$

The first derivative of the hyperbolic tangent function has the following property [35]:

$$
\frac{\partial f(x)}{\partial x}=1-f(x)^{2}
$$

Hence, using such an expression and deriving with respect to a generic $j$ th activation value of the hidden layer, it follows that

$$
\frac{\partial y_{i k}}{\partial a_{j}^{(1)}}=\frac{\partial w_{j k}^{(2)} f\left(a_{j}^{(1)}\right)}{\partial a_{j}^{(1)}}=w_{j k}^{(2)} \frac{\partial f\left(a_{j}^{(1)}\right)}{\partial a_{j}^{(1)}}=w_{j k}^{(2)}\left[1-f\left(a_{j}^{(1)}\right)^{2}\right] \text {, }
$$

The second partial derivative instead can be expressed as

$$
\frac{\partial a_{j}^{(1)}}{\partial w_{s j}^{(1)}}=\frac{\partial w_{s j}^{(1)} x_{i s}}{\partial w_{s j}^{(1)}}=x_{i s} \text {. }
$$

Therefore, by rearranging these equations, the final value of the partial derivative of the $E_{i}$ component with respect to a weight of the hidden layer and of the output layer can be written as

$$
\begin{aligned}
& \frac{\partial E_{i}}{\partial w_{s j}^{(1)}}=\sum_{k=1}^{c}\left(y_{i k}-t_{i k}\right) w_{j k}^{(2)}\left[1-f\left(a_{j}^{(1)}\right)^{2}\right] x_{i s}, \\
& \frac{\partial E_{i}}{\partial w_{j k}^{(2)}}=\left(y_{i k}-t_{i k}\right) f\left(a_{j}^{(1)}\right) .
\end{aligned}
$$

In this way, it is possible to evaluate the gradient and to optimize the weight values at each iteration.

2.4. Training Algorithm. The training algorithm adopted in the current study was similar to that of the gradient descent, but slightly modified; it was the backpropagation algorithm of Levenberg-Marquardt [37]. Such an algorithm is of the second order but does not require the calculation of the Hessian matrix, which is approximated as

$$
\mathbf{H}=\mathbf{J}^{\mathrm{T}} \mathbf{J},
$$

where $\mathbf{H}$ is the Hessian matrix and $\mathbf{J}$ is the Jacobian matrix that contains the first derivatives of errors $\left(E_{i}\right)$ with respect to weights. The gradient $\mathbf{g}$ is instead calculated as

$$
\mathbf{g}=\mathbf{J}^{\mathrm{T}} \mathbf{e},
$$

where $\mathbf{e}$ is the vector of network errors. The Jacobian matrix can be calculated through the equations described above. The values of the weights are updated according to an iterative procedure similar to that of the gradient descent but modified as follows:

$$
\overline{\mathbf{w}}_{\tau}=\overline{\mathbf{w}}_{\boldsymbol{\tau}-1}-\mathbf{J}^{\mathrm{T}} \mathbf{e}\left[\mathbf{J}^{\mathrm{T}} \mathbf{J}+\mu \mathbf{I}\right]^{-1},
$$

TABLE 1: Diabase aggregates characteristic properties.

\begin{tabular}{lc}
\hline Property & Value \\
\hline Los Angeles coefficient (\%) & 25 \\
EN 1097-2 & 55 to 60 \\
Polished stone value (\%) & \\
EN 1097-8 & $<25$ \\
Flakiness index (\%) & \\
EN 933-3 & $>55$ \\
Equivalent in sand (\%) & \\
EN 933-8 & $<10$ (range of values 6.7 to 8.3) \\
Methylene blue value (mg/g) & \\
EN 933-9
\end{tabular}

TABLE 2: Bitumen characteristic properties.

\begin{tabular}{lcc}
\hline \multirow{2}{*}{ Property } & \multicolumn{2}{c}{ Bitumen type } \\
& $50 / 70$ & SBS modified \\
\hline $\begin{array}{l}\text { Penetration }(0.1 \times \mathrm{mm}) \\
\text { EN 1426 }\end{array}$ & 64 & 45 \\
Softening point $\left({ }^{\circ} \mathrm{C}\right)$ & 45.6 & 78.8 \\
EN 1427 & & \\
Elastic recovery $(\%)$ & - & 97.5 \\
EN 13398 & -7.0 & -15.0 \\
Fraas breaking point $\left({ }^{\circ} \mathrm{C}\right)$ & & \\
EN 12593 & - & 84 \\
After aging & - & -2.4 \\
Retained penetration $(\%)$ & & \\
Difference in softening point $\left({ }^{\circ} \mathrm{C}\right)$ & - & \\
\hline
\end{tabular}

where I is the identity matrix. It can be observed that if the scalar $\mu$ increases, it returns to having the gradient descent algorithm with $\eta$ small; the parameter $\mu$ is changed at each iteration; in particular, it is reduced to speed up the convergence to the solution.

\section{Materials and Methods}

The type of asphalt mixture considered in the current study was dense asphalt concrete (AC) with diabase aggregates and conventional or modified bitumen. The AC mixtures came from three different projects carried out in Greece, having various bitumen contents and aggregate gradations. The production of some of the AC mixtures was carried out in the laboratory either as part of the mix design procedure or as part of stiffness testing of the design mixture. The rest of the ACs were produced into a stationary asphalt plant as final mixture production.

3.1. Aggregates. The diabase aggregates, depending on the project, came from three different quarries; their characteristic properties, as well as the test protocols used, are given in Table 1.

3.2. Bitumen. Two types of bitumen have been used in the current study, a 50/70 conventional bitumen and a SBS modified bitumen. The characteristic properties of the two bitumen types, along with the test protocols adopted, are reported in Table 2 . 


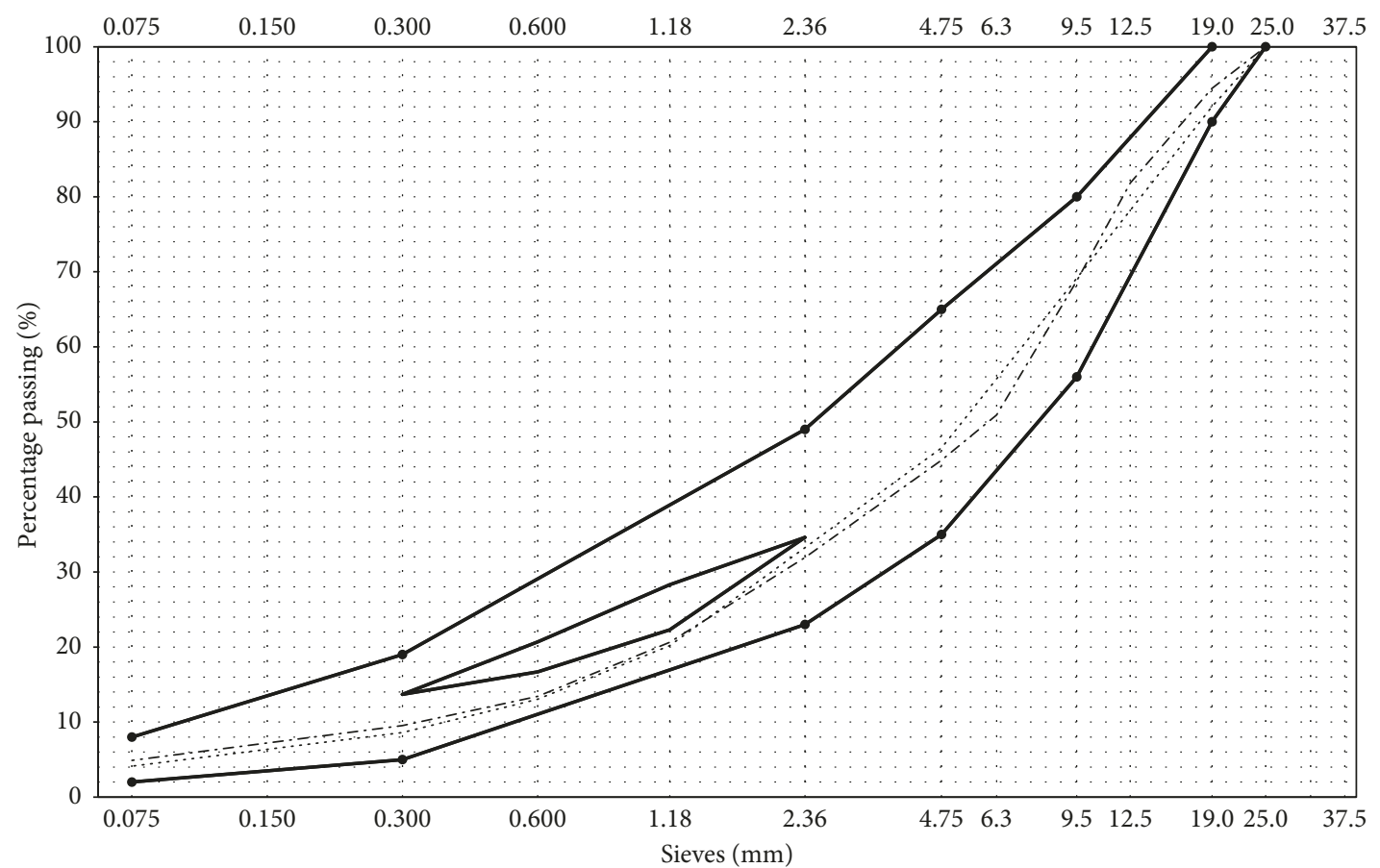

$\rightarrow$ Greek AC limits $\quad$..... AC20-50/70L

FIGURE 1: Gradation curves of AC20-50/70L and AC20-ModL.

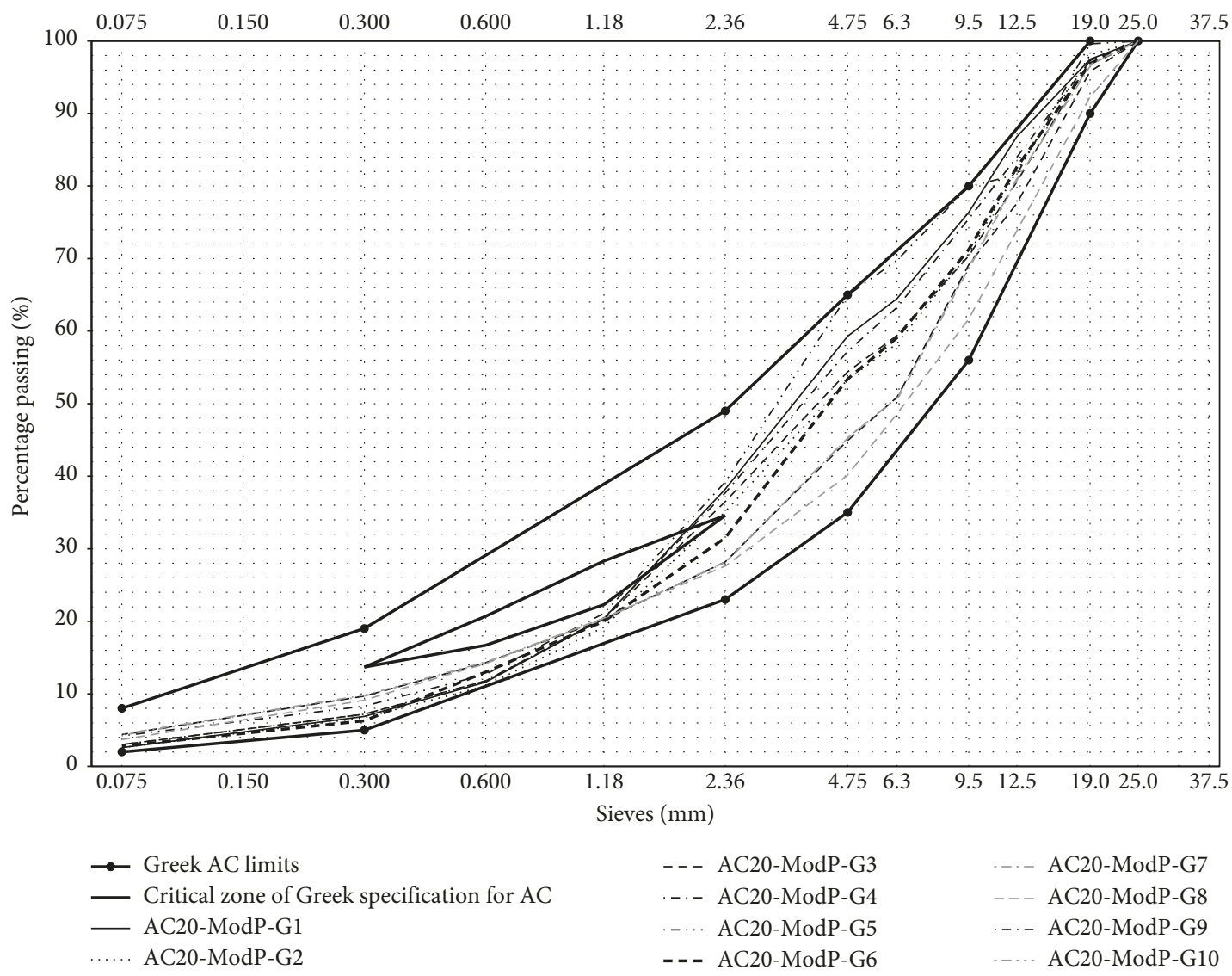

FIGURE 2: Gradation curves of AC20-ModP (gradations of different production dates). 
TABle 3: Properties of AC20-50/70L specimens.

\begin{tabular}{|c|c|c|c|c|c|c|}
\hline Specimen & $\begin{array}{l}\text { Bitumen content (\%) } \\
\text { (by weight of mix) }\end{array}$ & Voids (\%) & VMA (\%) & VFA (\%) & $\begin{array}{l}\text { Marshall stability } \\
(\mathrm{kN})\end{array}$ & Marshall flow (mm) \\
\hline AC20-50/70L-1 & 3.80 & 8.2 & 16.0 & 48.8 & 15.0 & 2.2 \\
\hline AC20-50/70L-2 & 3.80 & 8.0 & 15.9 & 49.3 & 14.6 & 2.3 \\
\hline AC20-50/70L-3 & 3.80 & 7.8 & 15.6 & 50.2 & 15.1 & 2.2 \\
\hline AC20-50/70L-4 & 3.80 & 8.4 & 16.2 & 48.0 & 15.5 & 2.2 \\
\hline AC20-50/70L-5 & 4.20 & 6.4 & 15.2 & 58.1 & 14.5 & 2.9 \\
\hline AC20-50/70L-6 & 4.20 & 6.1 & 14.9 & 59.4 & 14.5 & 2.6 \\
\hline AC20-50/70L-7 & 4.20 & 6.6 & 15.5 & 57.0 & 14.5 & 2.6 \\
\hline AC20-50/70L-8 & 4.20 & 6.9 & 15.7 & 56.1 & 13.9 & 2.7 \\
\hline AC20-50/70L-9 & 4.60 & 5.2 & 15.0 & 65.4 & 14.1 & 3.0 \\
\hline AC20-50/70L-10 & 4.60 & 5.9 & 15.7 & 62.1 & 13.4 & 3.1 \\
\hline AC20-50/70L-11 & 4.60 & 5.5 & 15.3 & 64.0 & 13.7 & 2.9 \\
\hline AC20-50/70L-12 & 4.60 & 6.3 & 16.0 & 60.7 & 14.4 & 3.2 \\
\hline AC20-50/70L-13 & 5.00 & 3.8 & 14.7 & 73.8 & 12.9 & 3.4 \\
\hline AC20-50/70L-14 & 5.00 & 3.8 & 14.6 & 74.1 & 12.8 & 3.3 \\
\hline AC20-50/70L-15 & 5.00 & 3.6 & 14.4 & 75.3 & 11.3 & 3.3 \\
\hline AC20-50/70L-16 & 5.00 & 3.8 & 14.7 & 73.9 & 13.1 & 3.2 \\
\hline AC20-50/70L-17 & 5.40 & 2.0 & 13.9 & 85.8 & 12.7 & 4.0 \\
\hline AC20-50/70L-18 & 5.40 & 2.1 & 14.0 & 84.9 & 12.0 & 4.2 \\
\hline AC20-50/70L-19 & 5.40 & 2.7 & 14.6 & 81.2 & 11.8 & 4.4 \\
\hline AC20-50/70L-20 & 5.40 & 2.4 & 14.3 & 83.0 & 12.0 & 4.5 \\
\hline AC20-50/70L-21 & 5.80 & 0.7 & 13.6 & 94.9 & 10.5 & 4.8 \\
\hline AC20-50/70L-22 & 5.80 & 0.5 & 13.5 & 96.4 & 10.9 & 4.7 \\
\hline AC20-50/70L-23 & 5.80 & 0.4 & 13.3 & 97.4 & 11.6 & 4.8 \\
\hline AC20-50/70L-24 & 5.80 & 0.5 & 13.5 & 96.3 & 11.2 & 5.0 \\
\hline AC20-50/70L-25 & 4.80 & 47 & 15.0 & 68.6 & 13.1 & 3.0 \\
\hline AC20-50/70L-26 & 4.80 & 4.8 & 15.1 & 68.1 & 12.7 & 3.2 \\
\hline AC20-50/70L-27 & 4.80 & 4.6 & 14.9 & 69.1 & 13.0 & 3.1 \\
\hline AC20-50/70L-28 & 4.80 & 5.0 & 15.3 & 67.3 & 13.0 & 3.2 \\
\hline AC20-50/70L-29 & 520 & 2.1 & 13.5 & 84.7 & 12.0 & 3.5 \\
\hline AC20-50/70L-30 & 5.20 & 2.3 & 13.7 & 83.3 & 11.9 & 3.6 \\
\hline
\end{tabular}

TABle 4: Properties of AC20-ModL specimens.

\begin{tabular}{|c|c|c|c|c|c|c|}
\hline Specimen & $\begin{array}{l}\text { Bitumen content (\%) } \\
\text { (by weight of mix) }\end{array}$ & Voids (\%) & VMA (\%) & VFA (\%) & Marshall stability $(\mathrm{kN})$ & Marshall flow (mm) \\
\hline AC20-ModL-1 & 4.20 & 6.1 & 14.1 & 57.1 & 9.9 & 2.3 \\
\hline AC20-ModL-2 & 4.20 & 6.0 & 14.1 & 57.2 & 8.9 & 1.9 \\
\hline AC20-ModL-3 & 4.20 & 6.4 & 14.4 & 55.8 & 9.8 & 2.3 \\
\hline AC20-ModL-4 & 4.60 & 4.1 & 13.3 & 69.2 & 10.2 & 3.0 \\
\hline AC20-ModL-5 & 4.60 & 4.2 & 13.3 & 68.8 & 9.3 & 3.1 \\
\hline AC20-ModL-6 & 4.60 & 4.7 & 13.8 & 66.1 & 11.9 & 3.1 \\
\hline AC20-Mod-7 & 5.00 & 3.6 & 13.7 & 74.1 & 10.5 & 3.0 \\
\hline AC20-ModL-8 & 5.00 & 4.3 & 14.4 & 70.0 & 10.2 & 3.9 \\
\hline AC20-ModL-9 & 5.00 & 3.5 & 13.7 & 74.2 & 12.4 & 3.6 \\
\hline AC20-ModL-10 & 5.60 & 1.3 & 13.1 & 90.4 & 12.7 & 5.0 \\
\hline AC20-ModL-11 & 5.60 & 2.3 & 14.0 & 83.7 & 11.6 & 3.5 \\
\hline AC20-ModL-12 & 5.60 & 2.4 & 14.2 & 82.7 & 11.7 & 4.2 \\
\hline AC20-ModL-13 & 4.60 & 3.3 & 12.3 & 73.4 & 9.6 & 3.9 \\
\hline AC20-ModL-14 & 4.60 & 3.0 & 12.1 & 75.4 & 9.6 & 3.1 \\
\hline AC20-ModL-15 & 4.60 & 3.7 & 12.7 & 71.2 & 12.1 & 3.1 \\
\hline AC20-ModL-16 & 5.00 & 3.8 & 13.7 & 72.6 & 10.6 & 3.0 \\
\hline AC20-ModL-17 & 5.00 & 4.5 & 14.4 & 68.5 & 11.1 & 3.9 \\
\hline AC20-ModL-18 & 5.00 & 4.8 & 14.6 & 67.4 & 12.2 & 3.6 \\
\hline AC20-ModL-19 & 4.9 & 4.8 & 15.7 & 69.5 & 13.3 & 3.6 \\
\hline AC20-ModL-20 & 4.90 & 4.4 & 15.4 & 71.1 & 13.2 & 3.5 \\
\hline AC20-ModL-21 & 4.90 & 3.6 & 14.6 & 75.4 & 13.3 & 3.7 \\
\hline AC20-ModL-22 & 3.80 & 8.2 & 16.0 & 48.8 & 15.0 & 2.2 \\
\hline AC20-ModL-23 & 3.80 & 8.0 & 15.9 & 49.3 & 14.6 & 2.3 \\
\hline
\end{tabular}


TABle 4: Continued.

\begin{tabular}{lcccccc}
\hline Specimen & $\begin{array}{c}\text { Bitumen content (\%) } \\
\text { (by weight of mix) }\end{array}$ & Voids (\%) & VMA (\%) & VFA (\%) & Marshall stability (kN) & Marshall flow (mm) \\
\hline AC20-ModL-24 & 3.80 & 7.8 & 15.6 & 50.2 & 15.1 & 15.5 \\
AC20-ModL-25 & 3.80 & 8.4 & 16.2 & 48.0 & 14.5 & 2.2 \\
AC20-ModL-26 & 4.20 & 6.4 & 15.2 & 58.1 & 14.5 & 2.2 \\
AC20-ModL-27 & 4.20 & 6.1 & 14.9 & 59.4 & 14.5 & 2.9 \\
AC20-ModL-28 & 4.20 & 6.6 & 15.5 & 57.0 & 13.9 & 2.6 \\
AC20-ModL-29 & 4.20 & 6.9 & 15.7 & 56.1 & 14.5 & 3.7 \\
AC20-ModL-30 & 4.20 & 6.1 & 14.9 & 59.4 & & \\
\hline
\end{tabular}

Table 5: Properties of AC20-ModP specimens.

\begin{tabular}{|c|c|c|c|c|c|c|c|}
\hline Specimen & $\begin{array}{l}\text { Bitumen content (\%) } \\
\text { (by weight of mix) }\end{array}$ & $\begin{array}{l}\text { Voids } \\
(\%)\end{array}$ & $\begin{array}{l}\text { VMA } \\
(\%)\end{array}$ & $\begin{array}{l}\text { VFA } \\
(\%)\end{array}$ & $\begin{array}{c}\text { Marshall } \\
\text { stability } \\
(\mathrm{kN})\end{array}$ & $\begin{array}{l}\text { Marshall } \\
\text { flow (mm) }\end{array}$ & $\begin{array}{l}\text { Gradation (refers to } \\
\text { gradations shown in } \\
\text { Figure 2) }\end{array}$ \\
\hline AC20-ModP-1 & 5.22 & 7.5 & 19.5 & 61.4 & 13.3 & 3.2 & $\mathrm{G} 1$ \\
\hline AC20-ModP-2 & 5.22 & 7.2 & 19.2 & 62.3 & 12.7 & 3.3 & G1 \\
\hline AC20-ModP-3 & 5.22 & 8.1 & 20.0 & 59.3 & 11.7 & 4.4 & G1 \\
\hline AC20-ModP-4 & 4.89 & 7.7 & 18.8 & 59.0 & 12.8 & 3.8 & G2 \\
\hline AC20-ModP-5 & 4.89 & 8.8 & 19.8 & 55.5 & 11.2 & 4.1 & G2 \\
\hline AC20-ModP-6 & 4.89 & 9.1 & 20.1 & 54.5 & 9.4 & 3.5 & $\mathrm{G} 2$ \\
\hline AC20-ModP-7 & 4.49 & 8.9 & 18.9 & 52.9 & 11.4 & 3.3 & G3 \\
\hline AC20-ModP-8 & 4.49 & 9.0 & 19.0 & 52.5 & 11.9 & 3.6 & G3 \\
\hline AC20-ModP-9 & 4.49 & 9.7 & 19.6 & 50.5 & 10.6 & 3.4 & G3 \\
\hline AC20-ModP-10 & 4.86 & 8.9 & 19.8 & 55.0 & 11.3 & 3.4 & G4 \\
\hline AC20-ModP-11 & 4.86 & 8.2 & 19.1 & 57.4 & 11.4 & 3.1 & G4 \\
\hline AC20-ModP-12 & 4.86 & 8.8 & 19.7 & 55.5 & 12.6 & 2.7 & G4 \\
\hline AC20-ModP-13 & 4.72 & 7.7 & 19.5 & 60.5 & 10.1 & 5.2 & G5 \\
\hline AC20-ModP-14 & 4.72 & 8.4 & 20.2 & 58.1 & 8.2 & 5.4 & G5 \\
\hline AC20-ModP-15 & 4.72 & 8.7 & 20.4 & 57.4 & 7.8 & 5.2 & G5 \\
\hline AC20-ModP-16 & 4.81 & 7.7 & 19.7 & 61.2 & 10.9 & 5.0 & G6 \\
\hline AC20-ModP-17 & 4.81 & 7.2 & 19.3 & 62.7 & 10.5 & 5.4 & G6 \\
\hline AC20-ModP-18 & 4.81 & 7.2 & 19.3 & 62.6 & 8.1 & 5.4 & G6 \\
\hline AC20-ModP-19 & 4.40 & 5.8 & 17.1 & 64.7 & 13.9 & 3.2 & G7 \\
\hline AC20-ModP-20 & 4.40 & 5.5 & 16.9 & 70.0 & 14.3 & 3.6 & G7 \\
\hline AC20-ModP-21 & 4.40 & 6.1 & 17.3 & 63.4 & 13.2 & 4.1 & G7 \\
\hline AC20-ModP-22 & 4.92 & 5.5 & 18.1 & 65.3 & 15.7 & 3.8 & G8 \\
\hline AC20-ModP-23 & 4.92 & 4.6 & 17.3 & 76.1 & 14.9 & 3.9 & G8 \\
\hline AC20-ModP-24 & 4.92 & 5.2 & 17.8 & 70.7 & 12.3 & 3.7 & G8 \\
\hline AC20-ModP-25 & 4.92 & 5.6 & 18.2 & 73.6 & 11.8 & 3.5 & G9 \\
\hline AC20-ModP-26 & 4.92 & 6.5 & 19.0 & 59.6 & 10.9 & 3.9 & G9 \\
\hline AC20-ModP-27 & 4.92 & 5.2 & 17.9 & 73.7 & 11.4 & 4.2 & G9 \\
\hline AC20-ModP-28 & 5.04 & 4.6 & 17.7 & 75.6 & 16.5 & 3.8 & G10 \\
\hline AC20-ModP-29 & 5.04 & 5.0 & 18.0 & 72.9 & 14.6 & 3.9 & G10 \\
\hline AC20-ModP-30 & 5.04 & 5.1 & 18.1 & 70.5 & 14.0 & 3.5 & G10 \\
\hline
\end{tabular}

3.3. Asphalt Concretes. The dense asphalt concrete (AC) mixtures had a maximum aggregate size of $20 \mathrm{~mm}$ (AC20), in all cases. In detail, the AC20 mixture with 50/70 conventional bitumen (AC20-50/70L) was produced exclusively in the laboratory, while the AC20s with SBS-modified bitumen were produced in the laboratory (AC20-ModL) and in a stationary asphalt plant (AC20-ModP). The specimens of all mixtures were compacted in the laboratory using an impact compactor (EN 12697-30) having a diameter of $100 \mathrm{~mm}$ and an average thickness of $63.7 \mathrm{~mm}$. Thirty specimens for each type of mixture were produced; hence, total ninety specimens were utilized in the current study. The gradations of the AC20-50/70L and AC20-ModL are given in Figure 1. The gradations of the AC20-ModP are given in Figure 2 and correspond to different production dates throughout the project.

Tables 3-5 show specimens' volumetric properties (EN 12697-8), Marshall stability, and Marshall flow values (EN 12697-34), per type of mixture. Furthermore, the Marshall quotient has been computed per each specimen, equal to the ratio between Marshall stability and Marshall flow. Even if it has been previously outlined the partial representativity of the Marshall data with respect to the asphalt concrete behaviour, such a test is still widely adopted given the large experience 
TABle 6: Stiffness tests results.

\begin{tabular}{|c|c|c|c|c|c|}
\hline Specimen & $\begin{array}{l}\text { Stiffness modulus } \\
(\mathrm{MPa})\end{array}$ & Specimen & $\begin{array}{l}\text { Stiffness modulus } \\
(\mathrm{MPa})\end{array}$ & Specimen & $\begin{array}{l}\text { Stiffness modulus } \\
(\mathrm{MPa})\end{array}$ \\
\hline AC20-50/70L-1 & 4900 & AC20-ModL 1 & 5853 & AC20-ModP-1 & 5854 \\
\hline AC20-50/70L-2 & 4875 & AC20-ModL 2 & 5199 & AC20-ModP-2 & 5839 \\
\hline AC20-50/70L-3 & 4835 & AC20-ModL 3 & 5590 & AC20-ModP-3 & 5693 \\
\hline AC20-50/70L-4 & 4867 & AC20-ModL 4 & 5479 & AC20-ModP-4 & 5302 \\
\hline AC20-50/70L-5 & 4424 & AC20-ModL 5 & 5669 & AC20-ModP-5 & 5535 \\
\hline AC20-50/70L-6 & 4523 & AC20-ModL 6 & 5045 & AC20-ModP-6 & 4968 \\
\hline AC20-50/70L-7 & 4605 & AC20-ModL 7 & 4803 & AC20-ModP-7 & 5172 \\
\hline AC20-50/70L-8 & 4602 & AC20-ModL 8 & 4377 & AC20-ModP-8 & 5806 \\
\hline AC20-50/70L-9 & 4216 & AC20-ModL 9 & 3538 & AC20-ModP-9 & 5353 \\
\hline AC20-50/70L-10 & 4205 & AC20-ModL 10 & 4484 & AC20-ModP-10 & 5262 \\
\hline AC20-50/70L-11 & 4217 & AC20-ModL 11 & 5063 & AC20-ModP-11 & 5532 \\
\hline AC20-50/70L-12 & 4204 & AC20-ModL 12 & 4776 & AC20-ModP-12 & 5672 \\
\hline AC20-50/70L-13 & 3775 & AC20-ModL 13 & 5987 & AC20-ModP-13 & 4380 \\
\hline AC20-50/70L-14 & 3705 & AC20-ModL 14 & 5994 & AC20-ModP-14 & 4378 \\
\hline AC20-50/70L-15 & 3704 & AC20-ModL 15 & 5950 & AC20-ModP-15 & 4097 \\
\hline AC20-50/70L-16 & 3777 & AC20-ModL 16 & 2940 & AC20-ModP-16 & 5239 \\
\hline AC20-50/70L-17 & 3125 & AC20-ModL 17 & 2961 & AC20-ModP-17 & 4049 \\
\hline AC $20-50 / 70 L-18$ & 3100 & AC20-ModL 18 & 2930 & AC20-ModP-18 & 4049 \\
\hline AC $20-50 / 70 \mathrm{~L}-19$ & 3204 & AC20-ModL 19 & 5012 & AC20-ModP-19 & 5388 \\
\hline AC20-50/70L-20 & 3174 & AC20-ModL 20 & 5188 & AC20-ModP-20 & 5453 \\
\hline AC20-50/70L-21 & 2304 & AC20-ModL 21 & 5840 & AC20-ModP-21 & 5795 \\
\hline AC20-50/70L-22 & 2405 & AC20-ModL 22 & 5213 & AC20-ModP-22 & 5656 \\
\hline AC20-50/70L-23 & 2385 & AC20-ModL 23 & 5124 & AC20-ModP-23 & 5600 \\
\hline AC20-50/70L-24 & 2382 & AC20-ModL 24 & 5125 & AC20-ModP-24 & 5640 \\
\hline AC20-50/70L-25 & 4103 & AC20-ModL 25 & 5135 & AC20-ModP-25 & 5486 \\
\hline AC20-50/70L-26 & 4108 & AC20-ModL 26 & 4826 & AC20-ModP-26 & 5154 \\
\hline AC20-50/70L-27 & 4020 & AC20-ModL 27 & 4852 & AC20-ModP-27 & 5374 \\
\hline AC20-50/70L-28 & 4078 & AC20-ModL 28 & 4798 & AC20-ModP-28 & 5680 \\
\hline AC20-50/70L-29 & 3525 & AC20-ModL 29 & 4823 & AC20-ModP-29 & 5968 \\
\hline AC20-50/70L-30 & 3542 & AC20-ModL 30 & 4765 & AC20-ModP-30 & 5904 \\
\hline Average $(\mathrm{MPa})$ & 3830 & Average (MPa) & 4911 & Average (MPa) & 5309 \\
\hline $\begin{array}{l}\text { Standard deviation } \\
(\mathrm{MPa})\end{array}$ & 783 & $\begin{array}{c}\text { Standard deviation } \\
(\mathrm{MPa})\end{array}$ & 851 & $\begin{array}{c}\text { Standard deviation } \\
(\mathrm{MPa})\end{array}$ & 566 \\
\hline
\end{tabular}

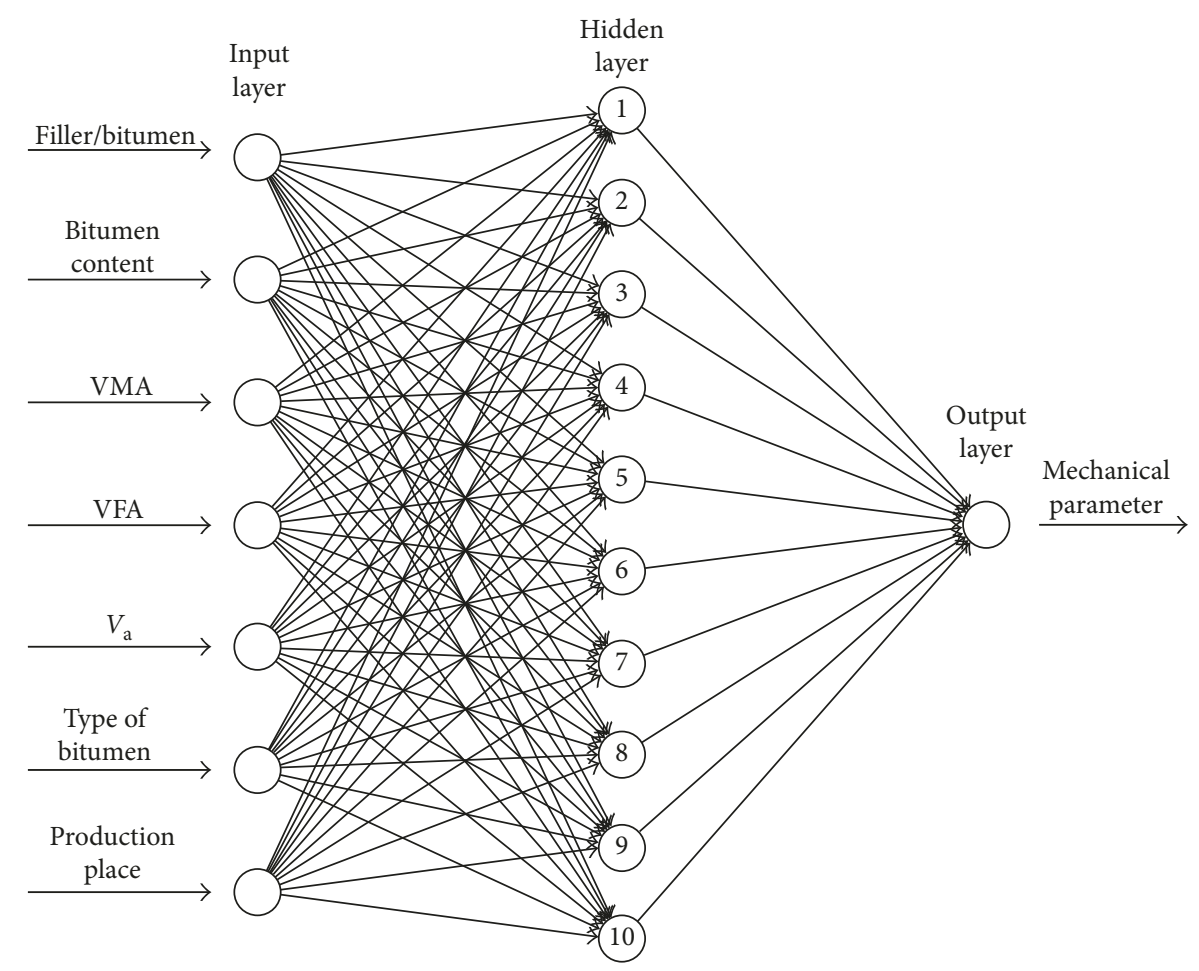

Figure 3: ANN structure adopted in the current study. 

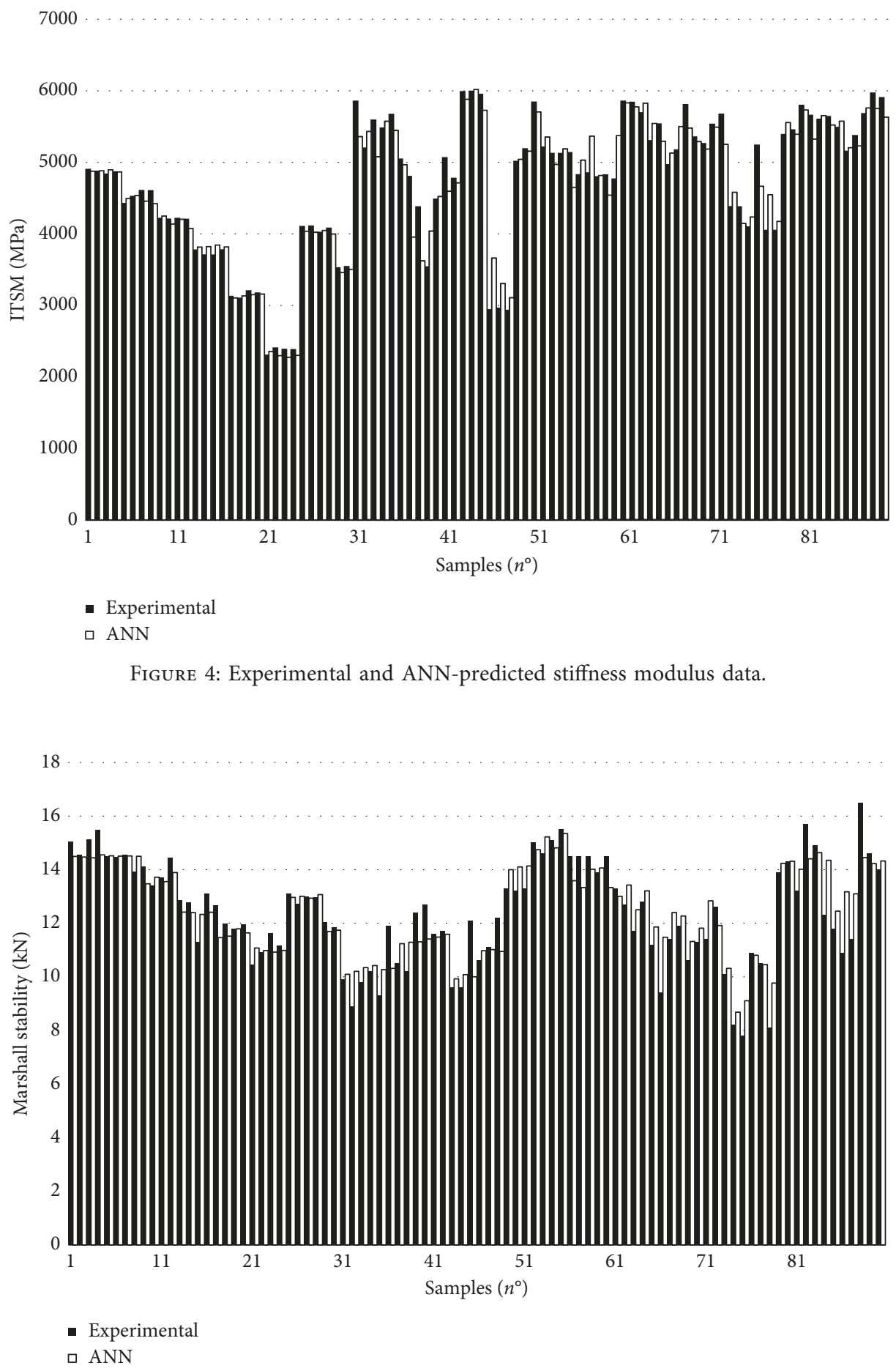

Figure 5: Experimental and ANN-predicted Marshall stability data.

cumulated over the years [38-41]. Table 5 gives additional details concerning the correspondence between specimens and gradations shown in Figure 2.

3.4. Stiffness Modulus Test. The Stiffness modulus has been evaluated, for all the specimens, in accordance with EN 12697-26, Annex C (IT-CY), assuming the following testing conditions: temperature of $20^{\circ} \mathrm{C}$, target deformation fixed at $5 \mu \mathrm{m}$, and rise-time equal to $124 \mathrm{~ms}$. The number of specimens tested for stiffness was ninety (90), that is, thirty (30) for each mixture, that is, AC20-50/70L, AC20-ModL, and AC20-ModP. The stiffness modulus results are presented in Table 6.

\section{Results and Discussion}

4.1. Stiffness Tests Results. Among the various experimental results, the stiffness tests deserve a dedicated discussion. As it 

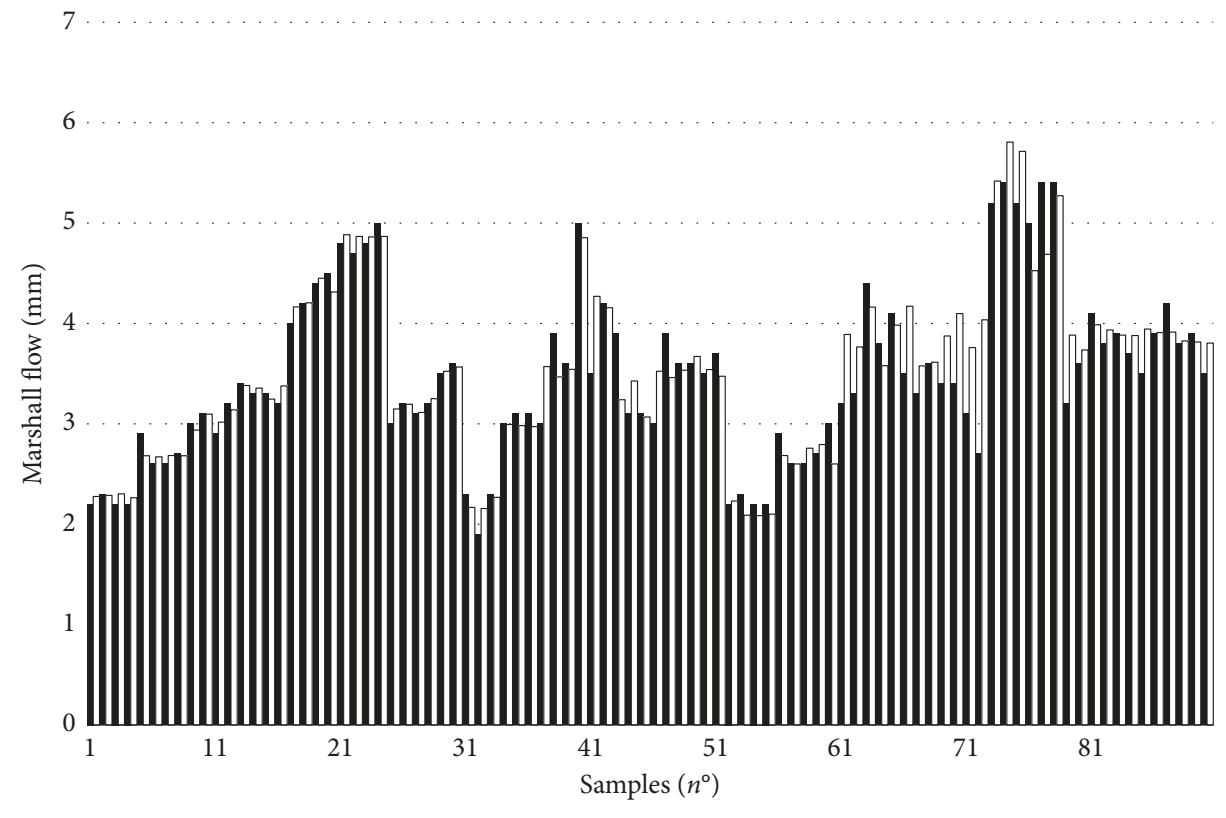

- Experimental

$\square$ ANN

Figure 6: Experimental and ANN-predicted Marshall flow data.

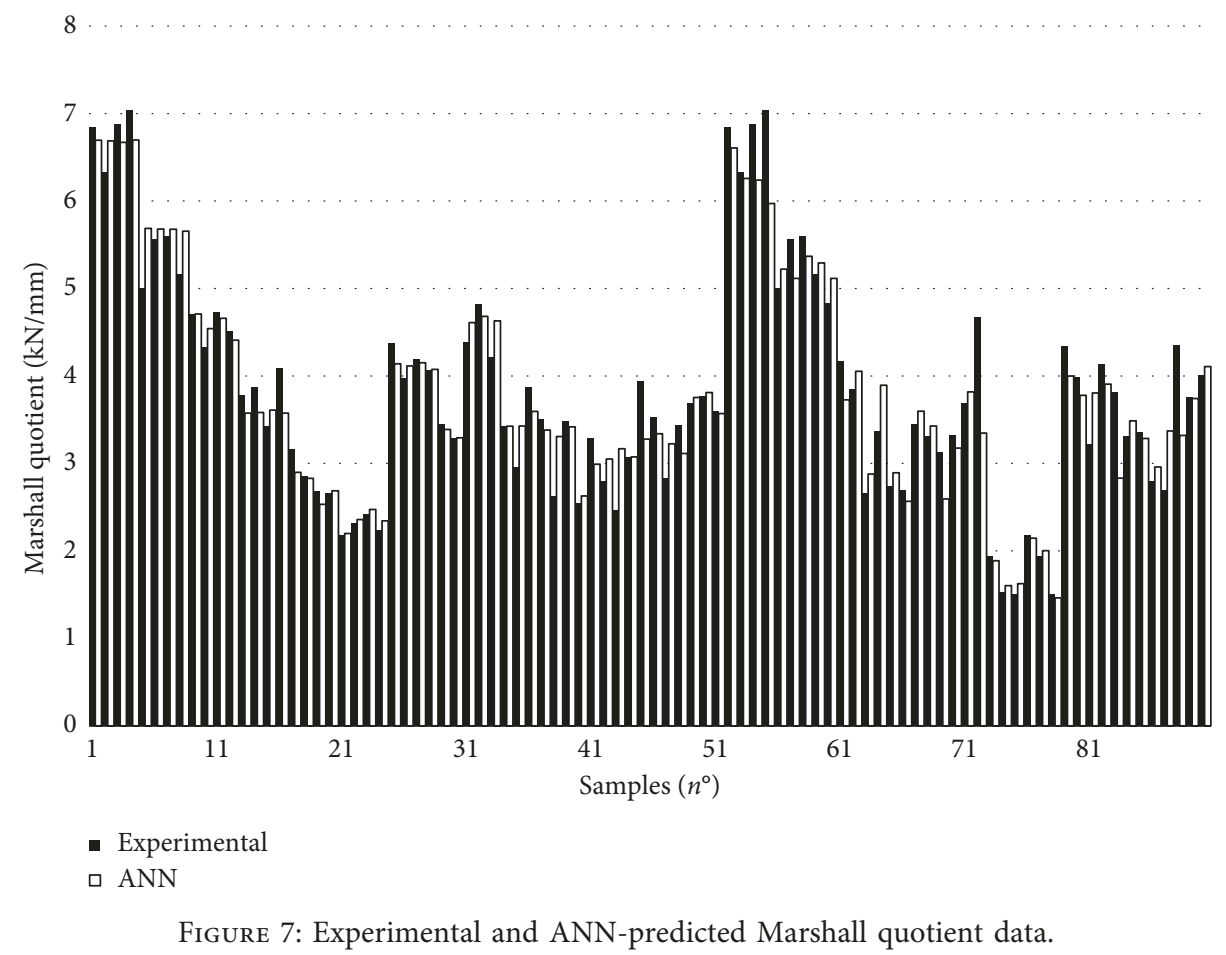

can be seen from Table 6, the AC mixtures with modified bitumen resulted in higher stiffness values than the AC mixtures with $50 / 70$ penetration bitumen. This can be attributed to the beneficial effect of the modified bitumen.

The smaller stiffness standard deviation was achieved for the plant-produced mixtures. This could be attributed to the machinery of the plant for asphalt production. More homogeneous mixtures are produced in a plant where large quantities are handled, and all production steps are automated, rather than in the laboratory. One of the most sensitive steps in laboratory production and specimen preparation is the filling of the Marshall moulds after asphalt mixing. Since 

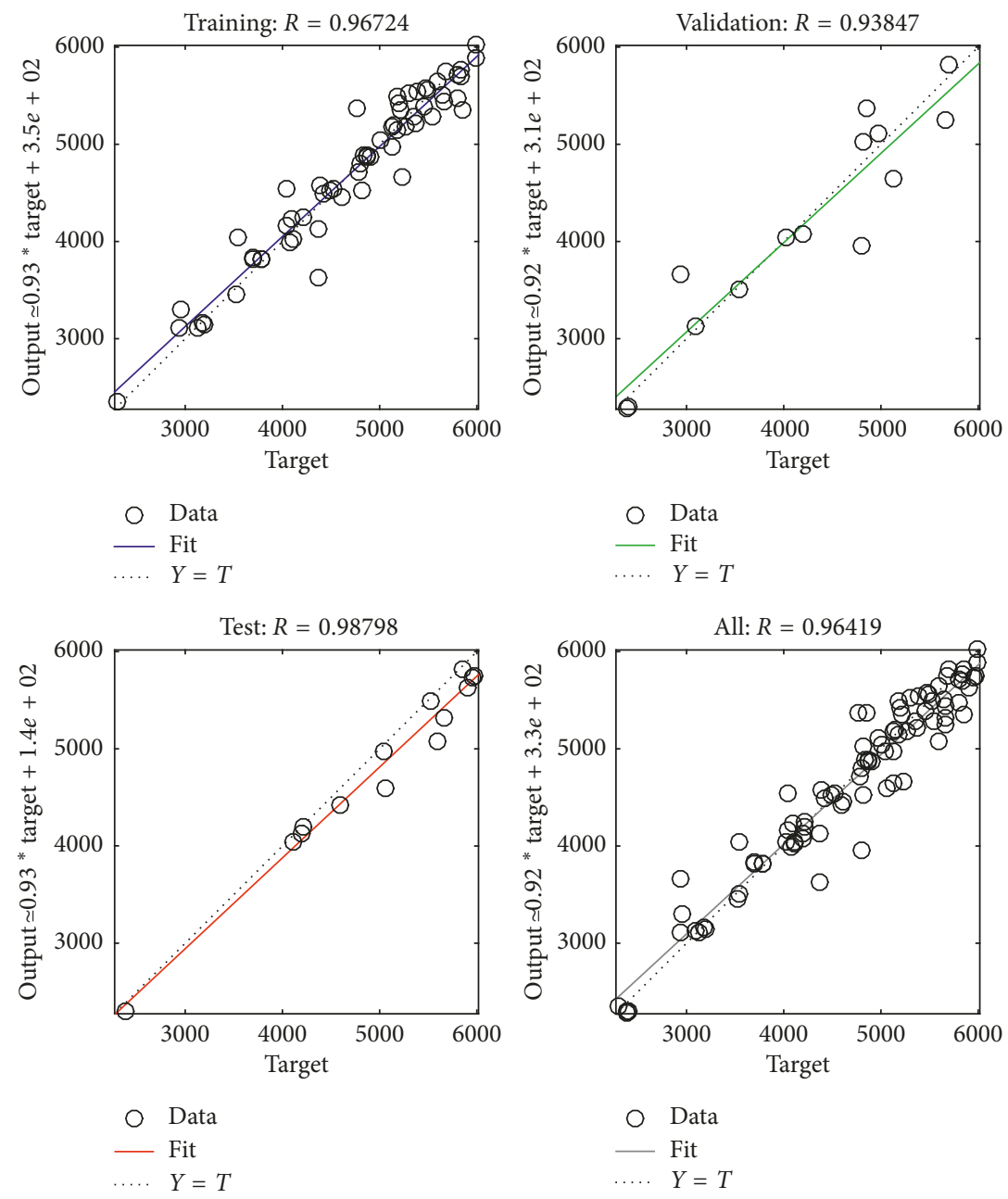

FIGURE 8: Training, validation, testing phases, and all data for the stiffness modulus (MPa) ANN model.

the mould filling is done by hand and the handler may not be the same in every case, it is normal not to have the same homogeneity obtained by automated plant procedures.

Considering each specimen stiffness value obtained in the current study, it could be stated that the stiffness values measured are depending mainly on the type of bituminous binder, bitumen content, and voids content. Gradation variations, especially for the plant-produced mixtures, are indirectly taken into account by the voids content.

4.2. Artificial Neural Networks Modelling Results. In the present study, a three-layer feedforward ANN was used to model each of the four mechanical parameters considered in the experimental investigation, namely, Marshall stability, flow and quotient, as well as Stiffness Modulus. The ANN models have been developed using the MATLAB ${ }^{\circledR}$ ANN toolbox [42].

The architecture of the four ANN models has been optimized with an input layer characterized by seven neurons, one hidden layer with 10 neurons, and an output layer with one neuron (Figure 3).

All the four ANN models have been elaborated on the basis of seven type of input data, namely, bitumen type, bitumen content, filler-bitumen ratio, air voids, voids in the mineral aggregates, voids filled with bitumen, and type of production process. Such input data have been considered of fundamental importance to take into account the different production process (laboratory or plant) and to properly represent the composition of the asphalt concretes, which were characterized by the same aggregate type, similar gradation, but prepared with different bitumen contents, binder type, and filler-bitumen ratios. Then, the single output neuron is associated with the specific mechanical parameter considered.

The number of neurons used in the hidden layer (10) has been optimized by means of a trial and error procedure; in the literature, it is recommended to use the lower number of neurons that allow us to obtain satisfactory results [32].

The experimental data set used for training and testing of the ANN has included 30 specimens for each of the three type of asphalt concrete (i.e., 90 specimens overall); therefore, all the mixtures, with modified or conventional bitumen, prepared in the laboratory or in the plant, have been analyzed together. This was done to obtain, for each of the four mechanical parameters considered, a unique predictive model, whatever the composition of the mixture, within the ambit of the current study. 

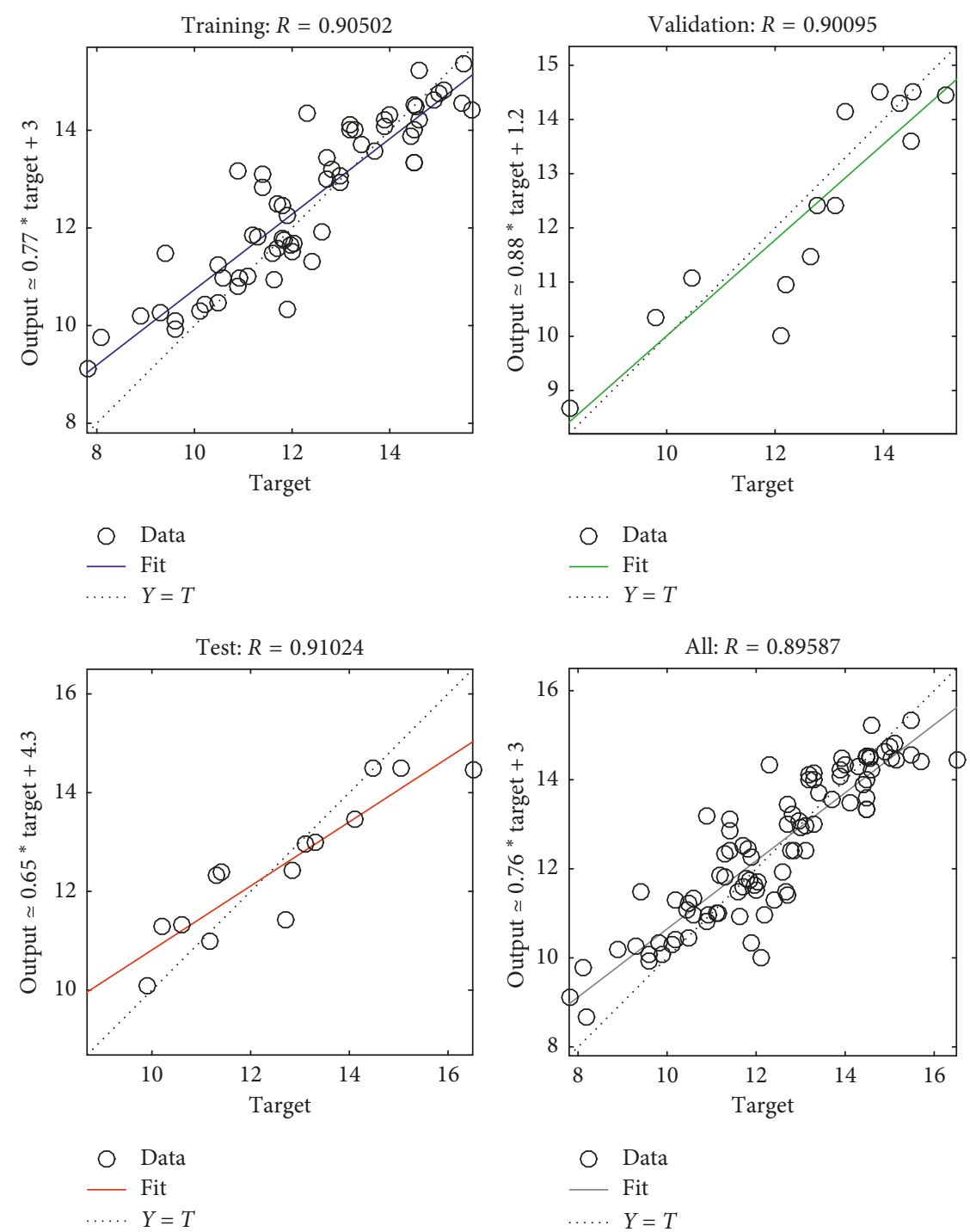

FIGURE 9: Training, validation, testing phases, and all data for the Marshall stability (kN) ANN model.

The $70 \%$ of the data set was used for the training of the ANN, a further $15 \%$ for the validation and the remaining $15 \%$ for the testing. The sampling process is completely random and is performed automatically by MATLAB [38].

Figures 4-7 show the comparison between experimental (target) and predicted (output) data, for the mechanical parameters analyzed. As it can be observed, the predicted values, computed by the ANN models, are very close to the experimental data, for both Marshall parameters and stiffness modulus. This is particularly significant form an engineering point of view, considering the different characteristics of the mixtures, in terms of composition and mechanical response.

The results of the training, validation, and testing phases of the ANN models can be observed in Figures 8-11.

In order to evaluate the performance of the ANN, two different statistical indicators are provided by MATLAB, namely, the coefficient of correlation $(R)$ and the mean square error (MSE) [42]; the closest to 1 the $R$ value and the lower the MSE value, the better the performance of the ANN. Similar performance indicators have been used in previous investigations, focused on the mechanical parameter prediction of asphalt concretes, by ANN analysis [20-22, 30-32]. The lowest MSE value (0.67751) was observed for the prediction of the stiffness modulus, while increasing values have been obtained for Marshall stability, flow, and quotient $(0.9962,1.6377$, and 1.6830, resp.). The highest accuracy achieved for the prediction of the stiffness modulus has been also confirmed in terms of correlation coefficient, with an $R$ value in the testing phase equal to 0.98798 . However, $R$ values greater than 0.91 were also obtained for the Marshall parameters (stability, flow, and quotient). Therefore, it can be concluded that the ANN approach allows us to obtain a satisfactory interpretation of the nonlinear relationships between the input variables considered and the mechanical parameters analysed.

Also previous studies have verified the good matching between experimental and ANN model results for stiffness [32] and Marshall stability [21]. Nevertheless, other researchers have outlined a worst prediction of Marshall flow and Marshall quotient, with respect to Marshall 

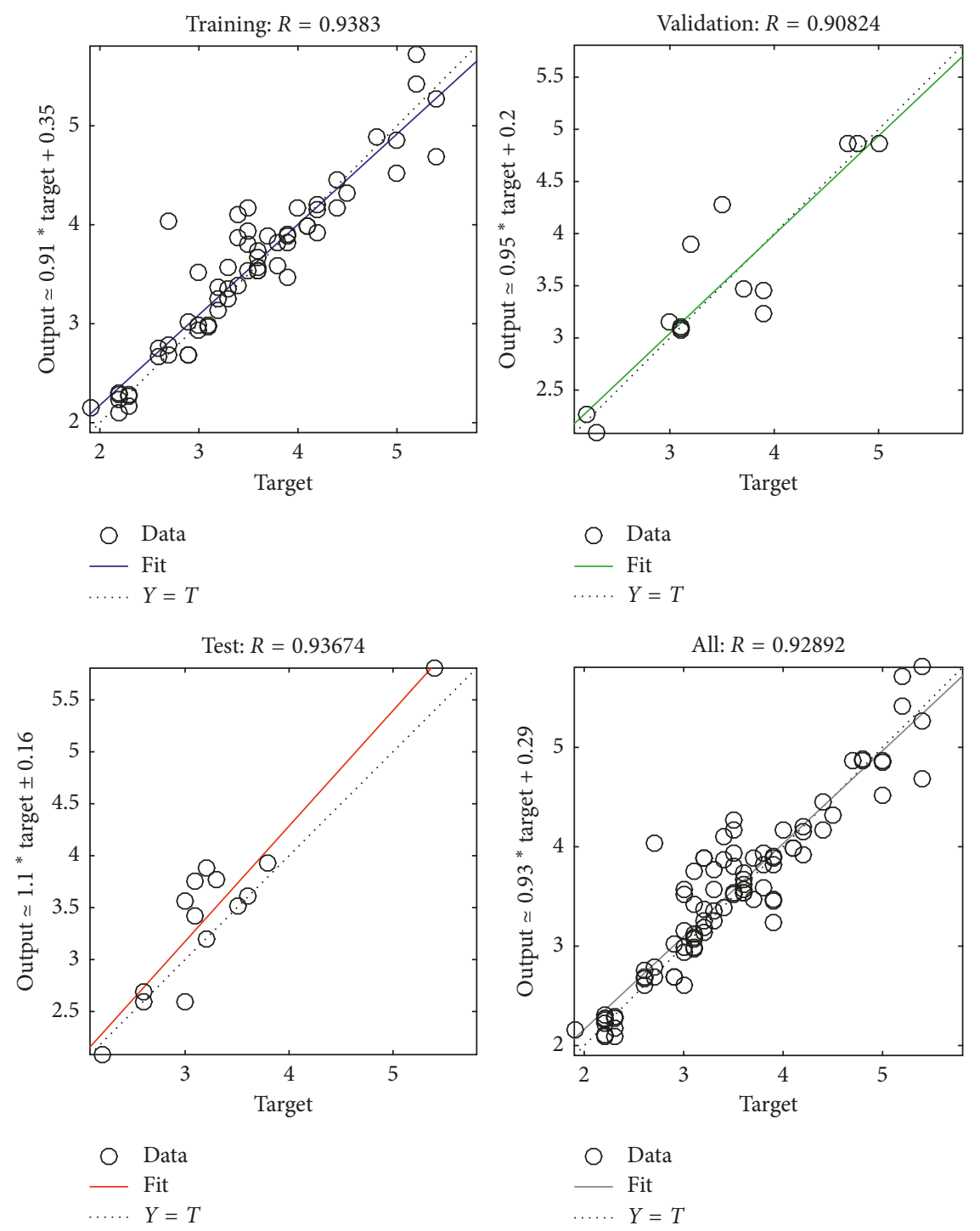

Figure 10: Training, validation, testing phases, and all data for the Marshall flow (mm) ANN model.

stability [20]. According to the results of the present study, all the Marshall parameters, as well as the stiffness modulus, have been predicted by the ANN models with a comparable quality level.
4.3. Predictive Equations. The general equation for the prediction of the mechanical parameter (MP) considered, obtained by the ANN modelization, is reported in the following:

$$
\operatorname{MP}\left(\frac{f}{b}, V_{\mathrm{a}}, \mathrm{VMA}, \mathrm{VFA}, b, T_{\mathrm{b}}, \mathrm{PP}\right)=\xi+\sum_{i=1}^{10} \frac{\varphi_{i}}{1+e^{\alpha_{i}-\beta_{i}(f / b)+\gamma_{i} V_{\mathrm{a}}+\delta_{i} \mathrm{VMA}+\varepsilon_{i} \mathrm{VFA}+\zeta_{i} b+\eta_{i} T_{\mathrm{b}}+\theta_{i} \mathrm{PP}}}
$$

where $f$ and $b$ are the filler and the bitumen contents, respectively; $V_{\mathrm{a}}$, VMA, and VFA are the residual air voids, voids in the mineral aggregate, and voids filled with bitumen; $T_{\mathrm{b}}$ is the type of bitumen; and PP is the production process type. The other ANN coefficients are reported in Tables 7-10, for the stiffness modulus and Marshall stability, flow, and quotient, respectively. The structure of the equation is characterized by the contribution of the ten artificial neurons, by means of the ten ANN coefficients.

Such a predictive model can be very useful to obtain an analytical estimation of the mechanical parameters analyzed, without the necessity to perform further experimental tests. This approach allows us to identify more easily and quickly the best mix design option, 

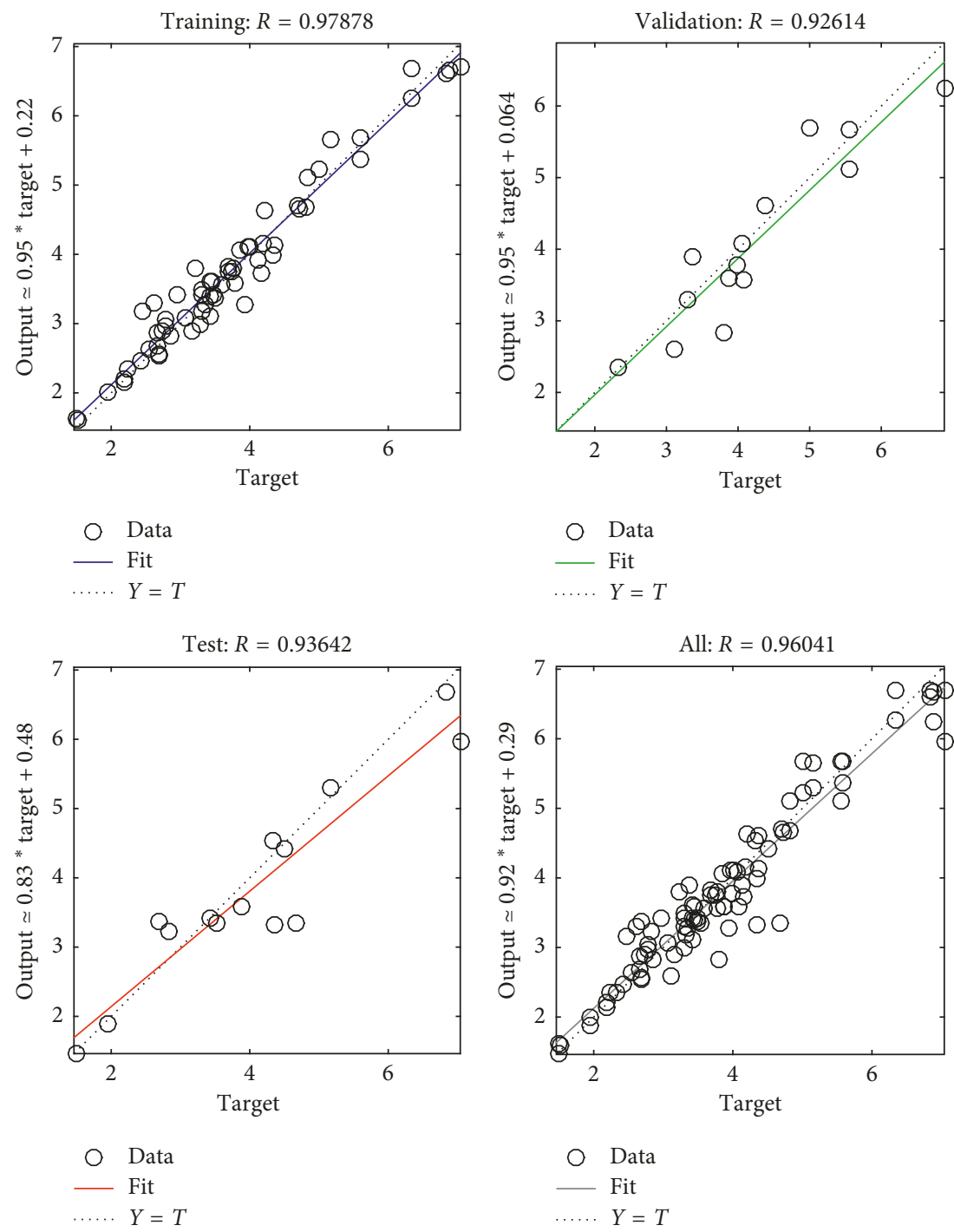

FIgURE 11: Training, validation, testing phases, and all data for the Marshall quotient $(\mathrm{kN} / \mathrm{mm})$ ANN model.

saving time and resources with respect to a direct laboratory evaluation.

\section{Conclusions}

In the present study, the artificial neural network approach has been used to numerically model the mechanical behaviour of asphalt concretes for road constructions.

The mixtures involved in the study were characterized by two different types of bituminous binder and production processes, as well as various bitumen contents and consequently different volumetric properties.

A feedforward multilayer ANN architecture, characterized by ten neurons in the hidden layer, has been elaborated to predict the stiffness modulus and the Marshall parameters of the mixes considered in the experimental investigation.

The hyperbolic tangent transfer function and a linear one have been assumed for the hidden and the output layers, respectively, whereas the Levenberg-Marquardt optimization algorithm was adopted as training algorithm.

The good results achieved in the testing phase demonstrate that the ANNs have the capability to generalize the complex relationships between input and output data, learned in the training phase, so allowing us to elaborate a satisfactory prediction model for the stiffness modulus, as well as for Marshall parameters, whatever the composition and the production process of the mixtures considered in the study.

A closed-form equation has been elaborated for each of the mechanical parameters studied, to allow other researchers and engineers to obtain an estimation of such parameters, within the type of mixes investigated.

The present study has demonstrated the feasibility to obtain, by means of ANN, predictive models of mechanical parameters (Marshall stability, flow, quotient, and stiffness modulus) very important for the mix design and the performance characterization of asphalt concretes for road pavements. However, even if different compositions of the 
Table 7: ANN coefficients for the stiffness modulus prediction model.

\begin{tabular}{|c|c|c|c|c|c|c|c|c|c|c|}
\hline \multirow{2}{*}{$\xi$} & \multirow{2}{*}{ Neurons } & \multicolumn{9}{|c|}{ ANN coefficients } \\
\hline & & $\varphi$ & $\alpha$ & $\beta$ & $\gamma$ & $\delta$ & $\varepsilon$ & $\zeta$ & $\eta$ & $\theta$ \\
\hline \multirow[t]{10}{*}{40409.52} & 1 & -912.11 & -6.99 & 0.25 & 0.33 & -0.31 & 0.01 & 3.22 & -5.13 & 2.75 \\
\hline & 2 & 6331.62 & 45.78 & -4.27 & -0.29 & 0.00 & -0.10 & 0.63 & -3.09 & -2.39 \\
\hline & 3 & 4915.93 & -22.47 & 2.55 & 0.18 & 0.54 & 0.04 & 3.11 & 4.28 & -3.84 \\
\hline & 4 & 3017.43 & -10.94 & 0.37 & -0.09 & 0.29 & 0.03 & -1.31 & -5.40 & 2.92 \\
\hline & 5 & -312.08 & -2.55 & 0.98 & 0.21 & 0.10 & -0.07 & 4.39 & -3.91 & 0.60 \\
\hline & 6 & 578.49 & -4.37 & -1.11 & 0.18 & -0.01 & -0.11 & 4.14 & 0.57 & 3.26 \\
\hline & 7 & -2776.52 & -11.04 & -2.50 & -0.14 & 0.23 & 0.00 & 5.76 & 4.61 & 1.82 \\
\hline & 8 & -6764.69 & -14.24 & -0.71 & -0.66 & 0.56 & 0.10 & 4.09 & 2.15 & -1.26 \\
\hline & 9 & -1786.82 & 6.43 & 0.29 & 0.58 & -0.47 & -0.03 & 1.38 & 1.97 & -0.69 \\
\hline & 10 & 6770.06 & -16.09 & 0.73 & -0.48 & -0.42 & 0.06 & -1.49 & 2.45 & 3.56 \\
\hline
\end{tabular}

TABle 8: ANN coefficients for the Marshall stability prediction model.

\begin{tabular}{|c|c|c|c|c|c|c|c|c|c|c|}
\hline \multirow{2}{*}{$\xi$} & \multirow{2}{*}{ Neurons } & \multicolumn{9}{|c|}{ ANN coefficients } \\
\hline & & $\varphi$ & $\alpha$ & $\beta$ & $\gamma$ & $\delta$ & $\varepsilon$ & $\zeta$ & $\eta$ & $\theta$ \\
\hline \multirow[t]{10}{*}{128.43} & 1 & -8.77 & -34.19 & 1.74 & 0.11 & -0.56 & -0.01 & 1.92 & 2.67 & 7.22 \\
\hline & 2 & -0.68 & -13.21 & 1.02 & -0.55 & -0.35 & -0.01 & 5.05 & 6.03 & 2.48 \\
\hline & 3 & 0.63 & 1.78 & 2.33 & -0.58 & 0.44 & -0.17 & 5.38 & 0.11 & -3.59 \\
\hline & 4 & -4.25 & -71.15 & 1.28 & 0.14 & 1.06 & 0.04 & -7.00 & 5.56 & 12.38 \\
\hline & 5 & -7.08 & 20.27 & 1.72 & 0.11 & -0.17 & -0.08 & -5.23 & -0.47 & -4.63 \\
\hline & 6 & 14.56 & -13.93 & 3.31 & 0.39 & -1.02 & -0.12 & -3.16 & 3.17 & 5.80 \\
\hline & 7 & 5.93 & -8.36 & 0.66 & 0.32 & 0.59 & 0.09 & -3.84 & -3.07 & -1.39 \\
\hline & 8 & -1.54 & 1.68 & 4.46 & -0.24 & -1.21 & 0.00 & -9.52 & -5.23 & 5.36 \\
\hline & 9 & 8.70 & 37.32 & -2.38 & -0.38 & -0.29 & 0.01 & -0.68 & -6.25 & -3.96 \\
\hline & 10 & -2.78 & 9.28 & -2.49 & 0.43 & -0.25 & 0.00 & -2.81 & -5.09 & 3.25 \\
\hline
\end{tabular}

TABle 9: ANN coefficients for the Marshall flow prediction model.

\begin{tabular}{|c|c|c|c|c|c|c|c|c|c|c|}
\hline \multirow{2}{*}{$\xi$} & \multirow{2}{*}{ Neurons } & \multicolumn{9}{|c|}{ ANN coefficients } \\
\hline & & $\varphi$ & $\alpha$ & $\beta$ & $\gamma$ & $\delta$ & $\varepsilon$ & $\zeta$ & $\eta$ & $\theta$ \\
\hline \multirow[t]{10}{*}{32.70} & 1 & -1.74 & -21.08 & 0.83 & -0.18 & -0.29 & 0.09 & 2.76 & 3.40 & 1.33 \\
\hline & 2 & 2.40 & 4.88 & -0.91 & -0.03 & -0.80 & -0.04 & 4.51 & 2.49 & 3.06 \\
\hline & 3 & 0.95 & -3.20 & 2.20 & 0.11 & 0.36 & -0.11 & -7.09 & -1.92 & 0.12 \\
\hline & 4 & -2.92 & -42.06 & 2.15 & 0.06 & 0.76 & -0.04 & -2.41 & 3.44 & 4.79 \\
\hline & 5 & 1.00 & -18.71 & 2.74 & -0.20 & -0.03 & 0.04 & 5.72 & -1.44 & 0.31 \\
\hline & 6 & 3.59 & -4.16 & 0.08 & -0.06 & -0.48 & 0.08 & -4.44 & 2.86 & 1.83 \\
\hline & 7 & 4.08 & -10.43 & -0.21 & -0.44 & 0.37 & -0.09 & 3.00 & -0.26 & 3.86 \\
\hline & 8 & 3.02 & 29.36 & -2.77 & 0.50 & -0.50 & -0.06 & -0.21 & -4.07 & -0.39 \\
\hline & 9 & 0.71 & 18.74 & -1.40 & 0.24 & -0.43 & 0.00 & -6.08 & -1.01 & -0.74 \\
\hline & 10 & 0.17 & -15.74 & -1.18 & 0.01 & 0.16 & 0.09 & 5.56 & -1.29 & 1.34 \\
\hline
\end{tabular}

TABLE 10: ANN coefficients for the Marshall quotient prediction model.

\begin{tabular}{|c|c|c|c|c|c|c|c|c|c|c|}
\hline \multirow{2}{*}{$\xi$} & \multirow{2}{*}{ Neurons } & \multicolumn{9}{|c|}{ ANN coefficients } \\
\hline & & $\varphi$ & $\alpha$ & $\beta$ & $\gamma$ & $\delta$ & $\varepsilon$ & $\zeta$ & $\eta$ & $\theta$ \\
\hline \multirow[t]{10}{*}{44.71} & 1 & 19.58 & 1.48 & 2.02 & -0.22 & 0.00 & -0.17 & -3.89 & -2.57 & 1.59 \\
\hline & 2 & -4.70 & -9.24 & -2.39 & -0.19 & 0.89 & 0.00 & -2.07 & -1.52 & 4.19 \\
\hline & 3 & -0.78 & 40.84 & -3.64 & -0.11 & -0.20 & -0.19 & 9.28 & -1.77 & -2.44 \\
\hline & 4 & -7.96 & -42.51 & 5.98 & 0.17 & -0.09 & -0.08 & -0.67 & -2.79 & 5.66 \\
\hline & 5 & -1.53 & -35.96 & 4.65 & -0.48 & -0.09 & 0.07 & -7.57 & 4.85 & 2.89 \\
\hline & 6 & 4.27 & -42.21 & 2.10 & -0.30 & 0.35 & 0.03 & 14.17 & 4.42 & 1.91 \\
\hline & 7 & -0.79 & 13.57 & 1.02 & -0.14 & -0.29 & -0.25 & -2.19 & 6.67 & -1.45 \\
\hline & 8 & -3.73 & 22.45 & -2.62 & -0.66 & -0.10 & -0.10 & -1.97 & -5.24 & 3.55 \\
\hline & 9 & 1.81 & -3.00 & 0.50 & -0.10 & 0.26 & 0.16 & 2.23 & -3.70 & -2.68 \\
\hline & 10 & 6.95 & -4.93 & 1.01 & 0.73 & -0.01 & -0.06 & -4.69 & -6.15 & 5.16 \\
\hline
\end{tabular}


mixes have been studied, it would be very useful to increase the variability of the input parameters, for instance, considering different sources of the aggregates; this requires just a further new training of the ANN with additional experimental data.

\section{Data Availability}

All the data underlying the findings of the current paper are provided in the tables included in the manuscript.

\section{Conflicts of Interest}

The authors declare that they have no conflicts of interest.

\section{References}

[1] J. L. Feiteira Dias, L. G. Picado-Santos, and S. D. Capitão, "Mechanical performance of dry process fine crumb rubber asphalt mixtures placed on the Portuguese road network," Construction and Building Materials, vol. 73, pp. 247-254, 2014.

[2] Q. T. Liu and S. P. Wu, "Effects of steel wool distribution on properties of porous asphalt concrete," Key Engineering Materials, vol. 599, pp. 150-154, 2014.

[3] A. García, J. Norambuena-Contreras, M. Bueno, and M. N. Partl, "Influence of steel wool fibers on the mechanical, thermal, and healing properties of dense asphalt concrete," Journal of Testing and Evaluation, vol. 42, no. 5, article 20130197, 2014.

[4] A. R. Pasandín and I. Pérez, "Overview of bituminous mixtures made with recycled concrete aggregates," Construction and Building Materials, vol. 74, pp. 151-161, 2015.

[5] M. Zaumanis, R. B. Mallick, and R. Frank, "100\% hot mix asphalt recycling: challenges and benefits," Transportation Research Procedia, vol. 14, pp. 3493-3502, 2016.

[6] L. Wang, H. Gong, Y. Hou, X. Shu, and B. Huang, "Advances in pavement materials, design, characterisation, and simulation," Road Materials and Pavement Design, vol. 18, no. 3, pp. 1-11, 2017.

[7] E. Masad, L. Tashman, D. Little, and H. Zbib, "Viscoplastic modeling of asphalt mixes with the effects of anisotropy, damage and aggregate characteristics," Mechanics of Materials, vol. 37, no. 12, pp. 1242-1256, 2005.

[8] M. Giunta and A. A. Pisano, "One dimensional viscoelastoplastic constitutive model for asphalt concrete," Multidiscipline Modeling in Materials and Structures, vol. 2, no. 2, pp. 247-264, 2006.

[9] M. Pasetto and N. Baldo, "Numerical visco-elastoplastic constitutive modelization of creep recovery tests on hot mix asphalt," Journal of Traffic and Transportation Engineering, vol. 3, no. 5, pp. 390-397, 2016.

[10] S. M. J. G. Erkens, X. Liu, and A. Scarpas, "3D finite element model for asphalt concrete response simulation," International Journal of Geomechanics, vol. 2, no. 3, pp. 305-330, 2002.

[11] M. Costanzi and D. Cebon, "Generalized phenomenological model for the viscoelasticity of idealized asphalts," Journal of Materials in Civil Engineering, vol. 26, no. 3, pp. 399-410, 2014.

[12] M. Pasetto and N. Baldo, "Computational analysis of the creep behaviour of bituminous mixtures," Construction and Building Materials, vol. 94, pp. 784-790, 2015.
[13] A. C. Collop, G. R. McDowell, and Y. Lee, "Use of the distinct element method to model the deformation behavior of an idealized asphalt mixture," International Journal of Pavement Engineering, vol. 5, no. 1, pp. 1-7, 2004.

[14] A. Abbas, E. Masad, T. Papagiannakis, and A. Shenoy, "Micromechanical modelling of the viscoelastic behavior of asphalt mixtures using the discrete-element method," International Journal of Geomechanics, vol. 7, no. 2, pp. 131-139, 2007.

[15] G. Dondi, A. Simone, V. Vignali, and G. Manganelli, "Numerical and experimental study of granular mixes for asphalts," Powder Technology, vol. 232, pp. 31-40, 2012.

[16] S. H. Kim and N. Kim, "Development of performance prediction models in flexible pavement using regression analysis method," KSCE Journal of Civil Engineering, vol. 10, no. 2, pp. 91-96, 2006.

[17] A. Laurinavičius and R. Oginskas, "Experimental research on the development of rutting in asphalt concrete pavements reinforced with geosynthetic materials," Journal of Civil Engineering and Management, vol. 12, no. 4, pp. 311-317, 2006.

[18] P. K. Shukla and A. Das, "A re-visit to the development of fatigue and rutting equations used for asphalt pavement design," International Journal of Pavement Engineering, vol. 9, no. 5, pp. 355-364, 2008.

[19] A. S. M. Asifur Rahman, M. M. Mendez Larrain, and R. A. Tarefder, "Development of a nonlinear rutting model for asphalt concrete based on Weibull parameters," International Journal of Pavement Engineering, pp. 1-10, 2017.

[20] S. Tapkın, A. Çevik, and Ü. Usar, "Prediction of Marshall test results for polypropylene modified dense bituminous mixtures using neural networks," Expert Systems with Applications, vol. 37, no. 6, pp. 4660-4670, 2010.

[21] E. Ozgan, "Artificial neural network based modelling of the Marshall stability of asphalt concrete," Expert Systems with Applications, vol. 38, no. 5, pp. 6025-6030, 2011.

[22] G. H. Shafabakhsh, O. Jafari Ani, and M. Talebsafa, "Artificial neural network modeling (ANN) for predicting rutting performance of nano-modified hot-mix asphalt mixtures containing steel slag aggregates," Construction and Building Materials, vol. 85, pp. 136-143, 2015.

[23] N. Zavrtanik, J. Prosen, M. Tušar, and G. Turk, "The use of artificial neural networks for modeling air void content in aggregate mixture," Automation in Construction, vol. 63, pp. 155-161, 2016.

[24] S. Terzi, "Modeling the pavement serviceability ratio of flexible highway pavements by artificial neural networks," Construction and Building Materials, vol. 21, no. 3, pp. 590-593, 2007.

[25] M. Saltan and S. Terzi, "Modeling deflection basin using artificial neural networks with cross-validation technique in backcalculating flexible pavement layer moduli," Advances in Engineering Software, vol. 39, no. 7, pp. 588-592, 2008.

[26] U. Kırbaş and M. Karaşahin, "Performance models for hot mix asphalt pavements in urban roads," Construction and Building Materials, vol. 116, pp. 281-288, 2016.

[27] M. Mazari and D. D. Rodriguez, "Prediction of pavement roughness using a hybrid gene expression programmingneural network technique," Journal of Traffic and Transportation Engineering, vol. 3, no. 5, pp. 448-455, 2016.

[28] G. Sollazzo, T. F. Fwa, and G. Bosurgi, “An ANN model to correlate roughness and structural performance in asphalt pavements," Construction and Building Materials, vol. 134, pp. 684-693, 2017.

[29] F. Leiva-Villacorta, A. Vargas-Nordcbeck, and D. H. Timm, "Non-destructive evaluation of sustainable pavement technologies 
using artificial neural networks," International Journal of Pavement Research and Technology, vol. 10, no. 2, pp. 139147, 2017.

[30] S. Tapkın, A. Çevik, and Ü. Usar, "Accumulated strain prediction of polypropylene modified Marshall specimens in repeated creep test using artificial neural networks," Expert Systems with Applications, vol. 36, no. 8, pp. 11186-11197, 2009.

[31] S. Tapkın, A. Çevik, and Ş. Özcan, "Utilising neural networks and closed form solutions to determine static creep behaviour and optimal polypropylene amount in bituminous mixtures," Materials Research, vol. 15, no. 6, pp. 865-883, 2012.

[32] F. Xiao and S. N. Amirkhanian, "Artificial neural network approach to estimating stiffness behaviour of rubberized asphalt concrete containing reclaimed asphalt pavement," Journal of Transportation Engineering, vol. 135, no. 8, pp. 580-589, 2009.

[33] R. Reed and R. J. Marks, Neural Smithing: Supervised Learning in Feedforward Artificial Neural Networks, MIT Press, Cambridge, MA, USA, 1999.

[34] T. Rashid, Make Your Own Neural Network, CreateSpace Independent Publishing Platform, Scotts Valley, CA, USA, 2016.

[35] M. T. Hagan, H. B. Demuth, M. H. Beale, and O. De Jesús, Neural Network Design, Campus Pub, Lexington, KY, USA, 2014.

[36] W. McCulloch and W. Pitts, "A logical calculus of the ideas immanent in nervous activity," Bulletin of Mathematical Biophysics, vol. 5, no. 4, pp. 115-133, 1943.

[37] M. T. Hagan and M. Menhaj, "Training feed-forward networks with the Marquardt algorithm," IEEE Transactions on Neural Networks, vol. 5, no. 6, pp. 989-993, 1994.

[38] A. Patel, M. P. Kulkarni, S. D. Gumaste, P. P. Bartake, K. V. K. Rao, and D. N. Singh, "A methodology for determination of resilient modulus of asphaltic concrete," Advances in Civil Engineering, vol. 2011, Article ID 936395, 6 pages, 2011

[39] A. N. Bdour, Y. Khalayleh, and A. A. Al-Omari, "Assessing mechanical properties of hot mix asphalt with wire wool fibers," Advances in Civil Engineering, vol. 2015, Article ID 795903, 6 pages, 2015.

[40] L. Djakfar, H. Bowoputro, B. Prawiro, and N. Tarigan, "Performance of recycled porous hot mix asphalt with gilsonite additive," Advances in Civil Engineering, vol. 2015, Article ID 316719, 7 pages, 2015.

[41] N. Saboo and P. Kumar, "Performance characterization of polymer modified asphalt binders and mixes," Advances in Civil Engineering, vol. 2016, Article ID 5938270, 12 pages, 2016.

[42] The MathWorks Inc., MATLAB ${ }^{\circledR}, R 2014 b$, The MathWorks Inc., Natick, MA, USA, 2015. 


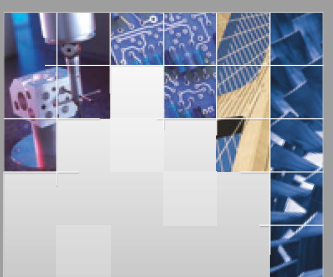

\section{Enfincering}
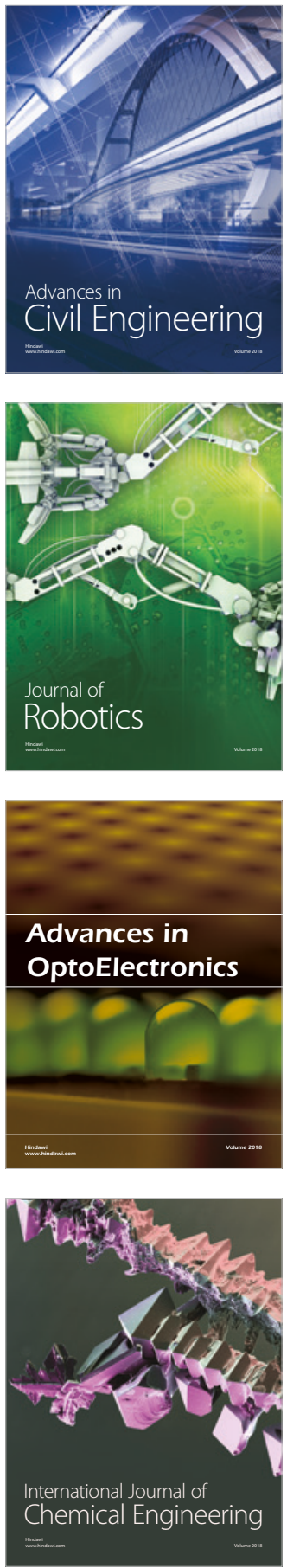

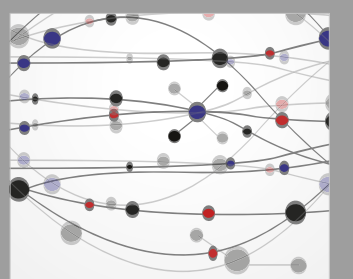

\section{Rotating \\ Machinery}

The Scientific World Journal

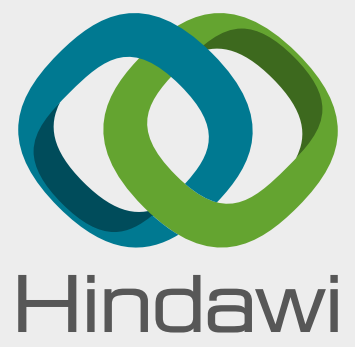

Submit your manuscripts at

www.hindawi.com
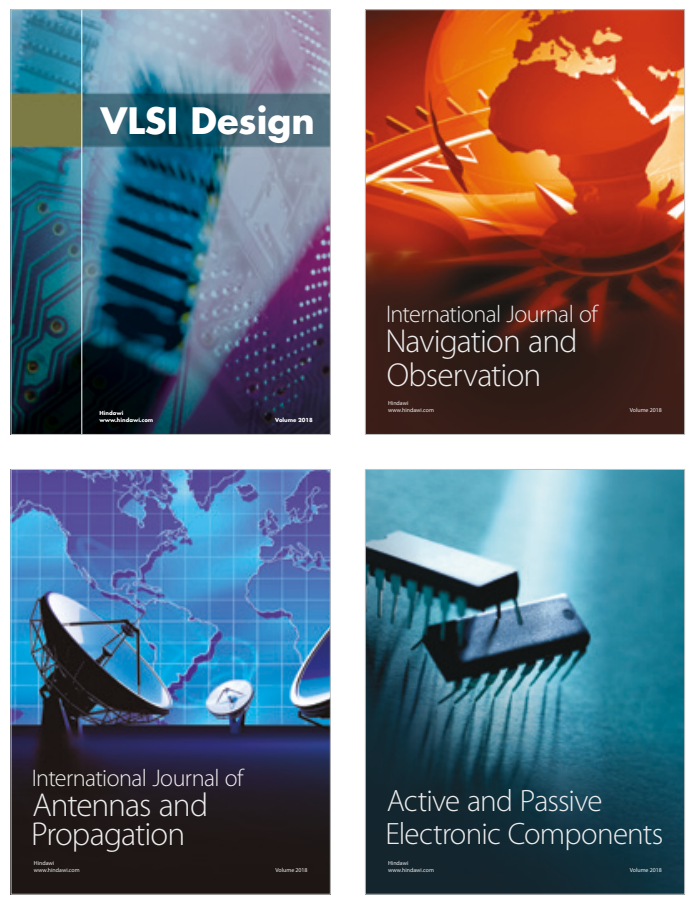
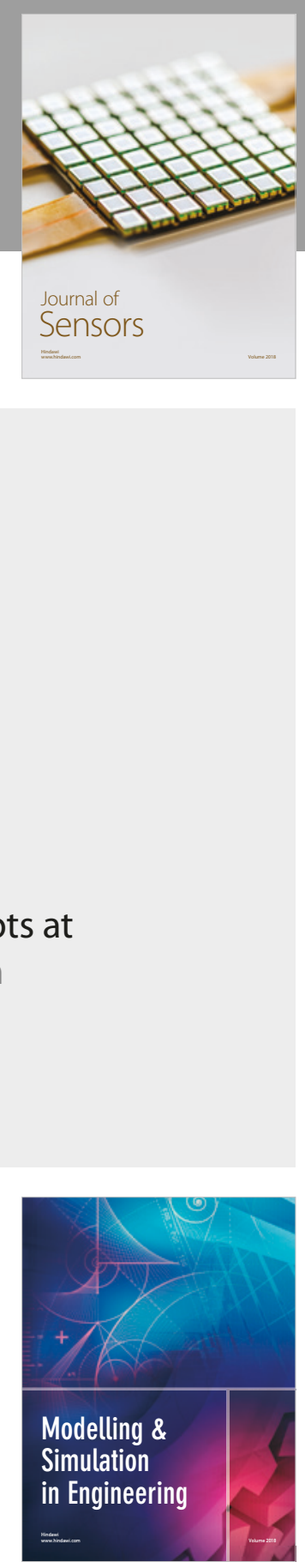

\section{Advances \\ Multimedia}
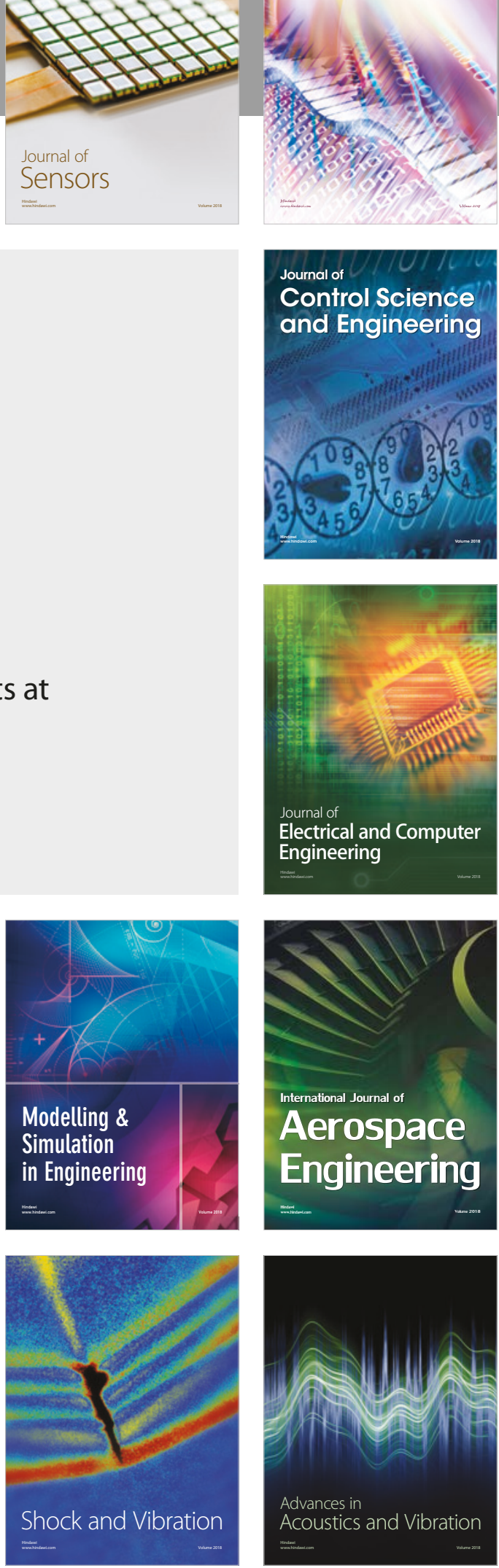